\title{
Computational and Experimental Unsteady Pressures for Alternate SLS Booster Nose Shapes
}

\author{
Gregory J. Brauckmann, Craig L. Streett† William L. Kleb† \\ Stephen J. Alter $\ddagger$ Kelly J. Murphy; and Christopher E. Glass ${ }^{\ddagger}$ \\ NASA Langley Research Center \\ Hampton, Virginia 23681
}

\begin{abstract}
Delayed Detached Eddy Simulation (DDES) predictions of the unsteady transonic flow about a Space Launch System (SLS) configuration were made with the Fully UNstructured Three-Dimensional (FUN3D) flow solver. The computational predictions were validated against results from a 2.5\% model tested in the NASA Ames 11-Foot Transonic Unitary Plan Facility. The peak $C_{p, r m s}$ value was under-predicted for the baseline, Mach 0.9 case, but the general trends of high $C_{p, r m s}$ levels behind the forward attach hardware, reducing as one moves away both streamwise and circumferentially, were captured. Frequency of the peak power in power spectral density estimates was consistently under-predicted. Five alternate booster nose shapes were assessed, and several were shown to reduce the surface pressure fluctuations, both as predicted by the computations and verified by the wind tunnel results.
\end{abstract}

\section{Nomenclature}

$\begin{array}{ll}a_{\infty} & \text { freestream speed of sound, ft/s } \\ C_{p} & \text { surface pressure coefficient } \\ C_{p, r m s} & \text { fluctuating component of } C_{p} \text { (root mean square) } \\ D & \text { core stage diameter, ft } \\ f & \text { frequency, Hz } \\ m & \text { number of time steps } \\ M_{\infty} & \text { freestream Mach number } \\ q_{\infty} & \text { freestream dynamic pressure, psi } \\ R e_{D} & \text { Reynolds number based on core stage diameter } \\ t & \text { time, sec } \\ \hat{t} & \text { time duration, sec } \\ T_{\infty} & \text { freestream static temperature, }{ }^{\circ} R \\ X & \text { streamwise geometric coordinate, in } \\ Y & \text { lateral geometric coordinate, in } \\ Z & \text { vertical geometric coordinate, in } \\ \alpha & \text { angle of attack, degrees } \\ \beta & \text { angle of sideslip, degrees } \\ \Delta f & \text { frequency resolution, Hz } \\ \Delta t & \text { time step, sec } \\ \Delta t^{\prime} & \text { non-dimensional time step } \\ \gamma & \text { specific heat ratio } \\ \phi & \text { circumferential angle (azimuth angle), degrees }\end{array}$

*Aerospace Research Engineer, Aerothermodynamics Branch, Associate Fellow AIAA

$\dagger$ Aerospace Research Engineer, Computational AeroSciences Branch, Senior Member AIAA

${ }^{\ddagger}$ Aerospace Research Engineer, Aerothermodynamics Branch, Senior Member AIAA 


\section{Introduction}

The Space Launch System (SLS) ${ }^{1,2}$ is a NASA program to develop a manned launch vehicle to replace the recently retired Space Transportation System (Space Shuttle). As the system matures, the current Shuttle-based solid-rocket-boosters (SRBs) may be replaced with advanced boosters, either solid or liquid fueled, that may have different geometrical shapes than the current boosters, such as larger diameters and blunter nose caps. A study was undertaken using computational fluid dynamic (CFD) tools to assess the aerodynamic impacts of these possible alternate booster designs, focusing on the prediction of the unsteady flow field that causes buffet. Buffet is a relatively low-frequency $(<100 \mathrm{~Hz})$ unsteady pressure loading that impacts the vehicle structural response as a whole, and is known to be a driver in the structural design of launch vehicles. ${ }^{3}$ Buffet forces are typically determined using sub-scale wind tunnel tests. ${ }^{4,5}$ The ability to computationally predict these flowfields and resultant loads, if realized, would provide a powerful capability to the SLS and future programs.

Computational resources have only occasionally been applied to buffet prediction in the past. One example is the case of the Titan IVB ${ }^{6}{ }^{6}$ where a laminar, unsteady Reynolds Averaged Navier-Stokes (RANS) approach was used to examine the flow downstream of the payload fairing. A more recent example is a paper analyzing the unsteady flow in the base region of the Ariane $5,{ }^{7}$ with emphasis on the three-dimensional effects due to the presence of side-mounted boosters. That work used a turbulent unsteady RANS approach with zonal detached eddy simulation (ZDES). The current work uses the Fully UNstructured Three-Dimensional (FUN3D) flow solver with a delayed detached eddy simulation (DDES), i.e., an integrated hybrid RANS/LES (Large Eddy Simulation) approach. This approach is more suitable to the present problem in that the flow in the wind tunnel will likely be turbulent, with a large wake behind the booster attach hardware, where a RANS approach would not be valid. The DDES approach does not require definition of a DES zone a priori, which is better suited to the unstructured grid environment of FUN3D.

The goal of this task is to establish the capability of assessing buffet for proposed geometries by using computational tools, in particular the FUN3D flow solver. CFD will not replace wind tunnel testing in the near term as the primary means of developing a buffet loads data base, especially for manned vehicles, but CFD can be used as an initial assessment and screening tool, to aid in wind tunnel test planning, and to provide insight into experimental results by providing more detailed flowfield analysis. The study was divided into two main parts: obtain a set of computational solutions on a series of booster nose shapes, and conduct a wind tunnel test for validation of the predictions. Modification of the booster nose shape was selected as the means to alter the vehicle flowfield around the booster forward attach point, a region identified as having elevated fluctuating pressure levels. A preliminary case had shown that the nose shape significantly affected the flow in the attach region, and changing only the booster nose, as opposed to wholesale swapping out of an entire booster, would be a relatively easy model change during a wind tunnel test. An arrangement had been made with the SLS program to piggyback on a previously scheduled aeroacoustic test, so minimal impact to that test's objectives was a primary consideration.

A companion paper by Alter et al. ${ }^{8}$ provides details of the FUN3D code and solution procedures, grid and time-step sensitivity analyses, and comparisons of a baseline case to wind tunnel results, while this paper will focus on the capability to use FUN3D to examine a broad array of booster nose shapes, and to examine the results in the frequency domain even with the limited (short duration) data sets.

Plots are shown with scales removed to mask absolute values of the data due to export control considerations.

\section{Geometry}

The basic geometry used in this investigation was the cargo variant of the proposed SLS launch vehicle. A layout is presented in Fig. 1a. The configuration consists of a central core, with an ogive nose and a cylindrical body, and two side-mounted cylindrical boosters with slightly blunted conical noses. Each booster is attached at two locations, one forward and one aft. Several geometric simplifications were made such as omitting vent lines, brackets, and booster separation motors. The azimuth or circumferential angles, shown in Fig. 1b, are defined as positive moving counter-clockwise from the $+\mathrm{Z}$ axis, such that $270^{\circ}$ for the core, and $90^{\circ}$ for the right-hand booster, face each other on the centerline between booster and core. 
Figure 2 shows the six noses. The baseline booster nose (Fig. 2a) is a spherically-blunted on-axis cone, designated as 'sharp on-axis'. Five alternate noses were analyzed: a canted version of the baseline nose (Fig. 2d), a blunt on-axis version (Fig. 2b), a canted version of the blunt nose (Fig. 2e), an on-axis ogive shape (Fig. 2c), and a canted ogive shape (Fig. 2f). All noses attached to the booster at the same vehicle station.

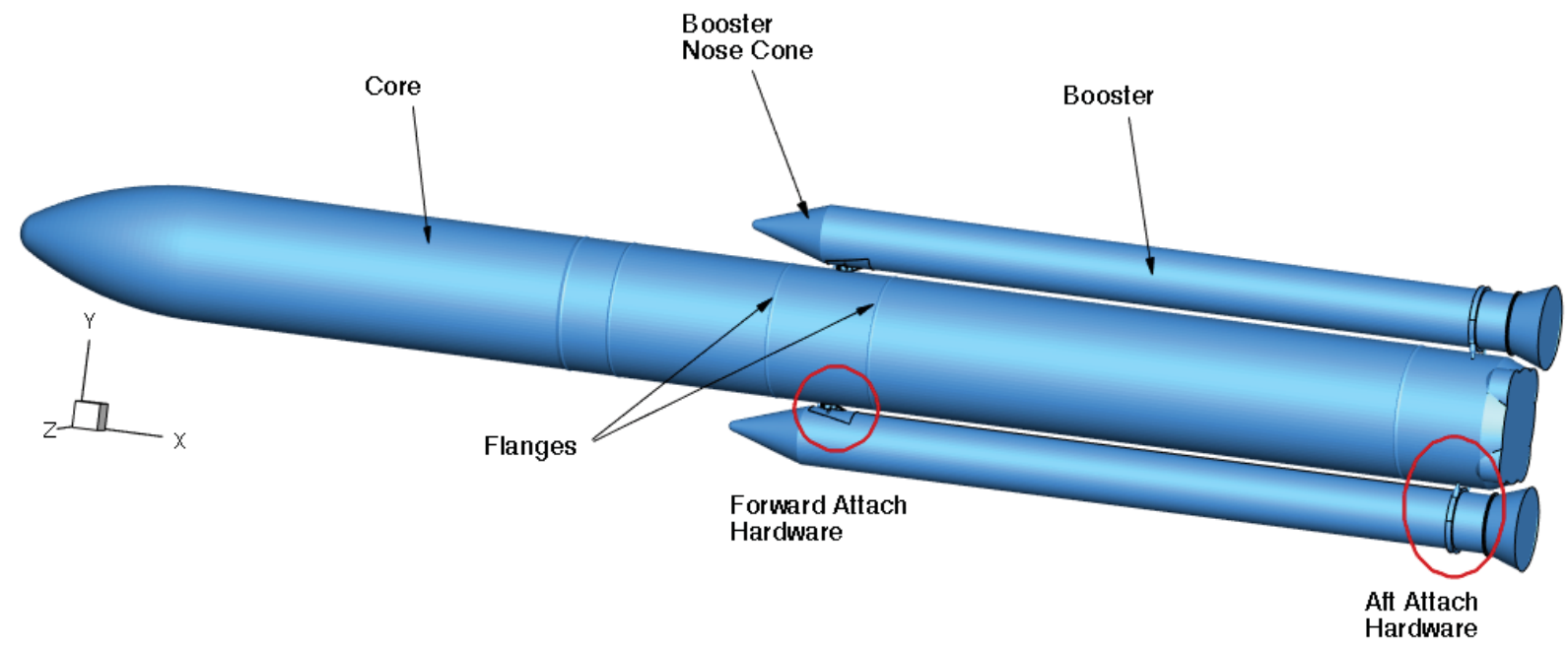

(a) General layout

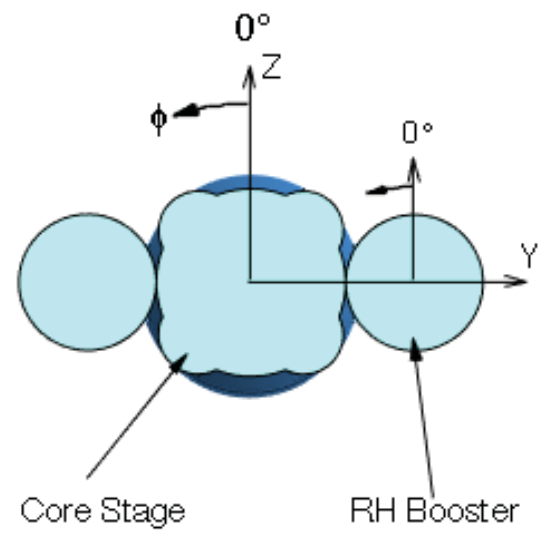

(b) Definition of azimuth angle

Figure 1: Layout of SLS cargo configuration.

\section{CFD Solver}

The FUN3D ${ }^{9}$ code was used to perform the CFD analyses presented in this paper. For this study, FUN3D was run using a modified Delayed Detached Eddy Simulation (DDES) ${ }^{10}$ hybrid RANS/LES model with a selectively-dissipative version of Edwards Low-Dissipation Flux Splitting Scheme (LDFSS) inviscid flux function, ${ }^{11}$ a modified Van Albada limiter, ${ }^{12}$ and a Spalart-Allmaras (SA) near-wall model. ${ }^{13}$ Nodebased conservative variables are computed by driving a 2nd-order accurate spatial residual to steady-state with a point-implicit iterative method. The solution was advanced in time with an optimal 2nd-order modified backward difference formula (BDF) scheme. ${ }^{14}$ Ideally, the solution would be advanced to the next time step when the mean flow residuals dropped an order of magnitude below the temporal error estimate. In practice, the solution was advanced when the ratio of mean flow to temporal errors was approximately unity. Additional details of the solution procedures can be found in the paper by Alter et al. ${ }^{8}$ 


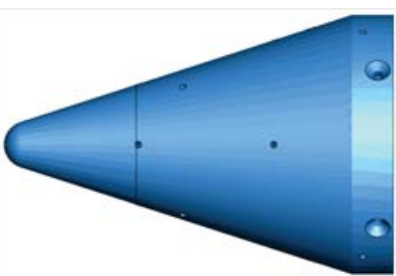

(a) Sharp on-axis

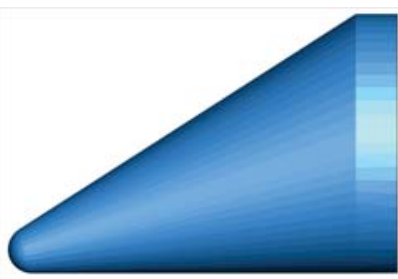

(d) Sharp canted

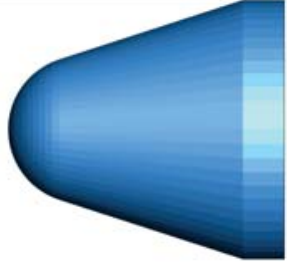

(b) Blunt on-axis

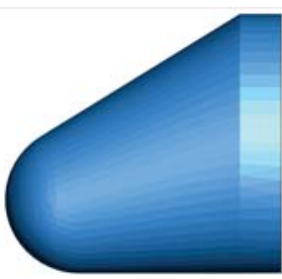

(e) Blunt canted

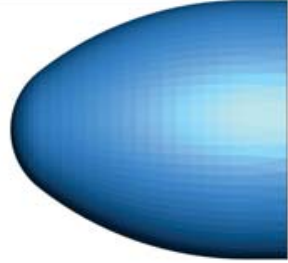

(c) Ogive on-axis

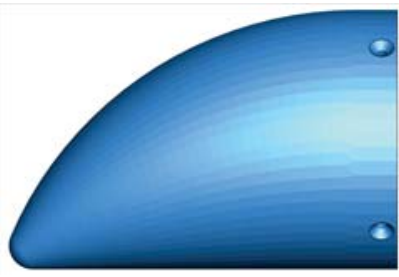

(f) Canted ogive

Figure 2: Baseline and alternate booster nose shapes.

All cases were run on a half-body domain, using only the right-hand side of the core but with a complete right-hand booster, as shown in Fig. 3a. Grid size was approximately 35 million nodes. Grid spacing varied over the geometry, with the densest grid around the forward attach hardware (Fig. 3b). A non-dimensional time step of 0.003 was used, as defined by Eq. 1,

$$
\Delta t^{\prime}=\Delta t \cdot a_{\infty} / D
$$

This value represents a physical time step of approximately $2 \mu \mathrm{s}$. The simulations were started impulsively, and the initial 15,000 time steps were discarded from the analyses to remove any start-up transients. Most cases were run an additional 45,000-55,000 time steps (0.087-0.106 seconds) past the startup transient. Several cases were run to much longer durations, with the baseline case computed for approximately 575,000 time steps, 1.105 seconds. Each of the shorter cases required approximately 300,000 core hours, or about 10 days on the $\mathrm{NAS}^{15}$ Pleiades system with 1,250 cores.

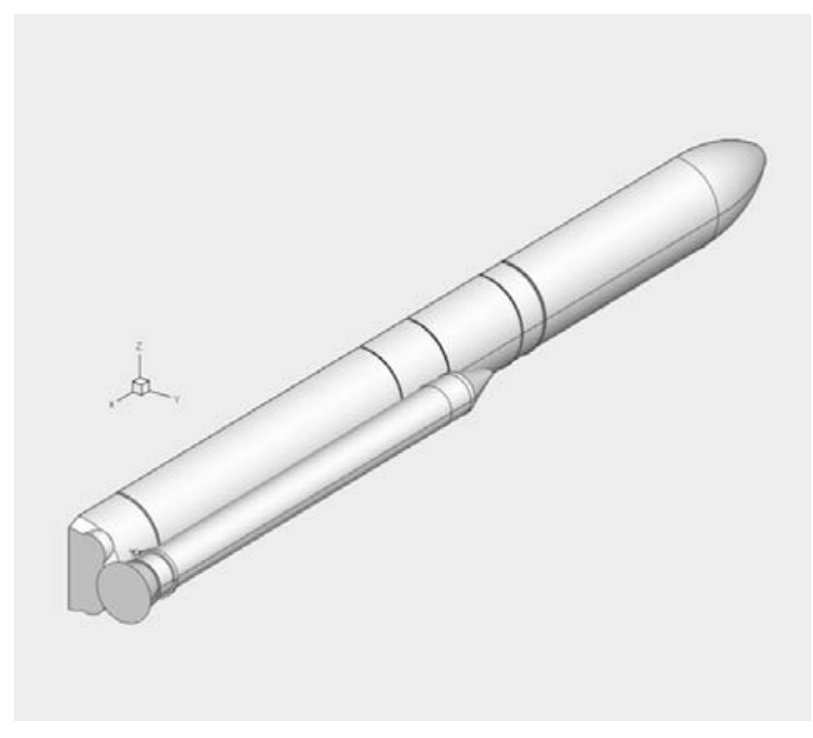

(a) half-body domain

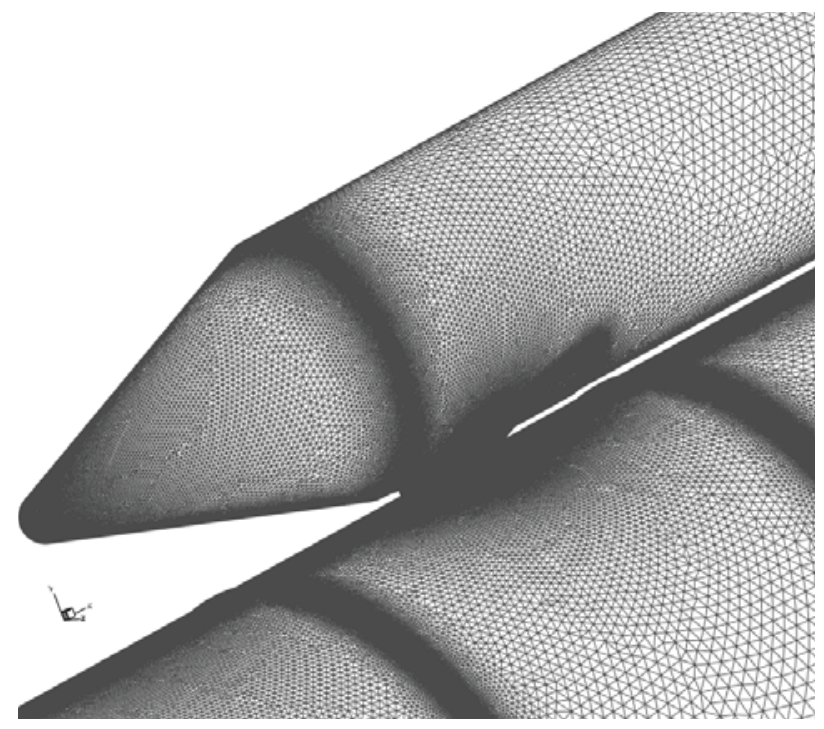

(b) close-up of grid in forward attach region

Figure 3: Computational geometry. 


\section{Cases}

Table 1 contains the CFD cases for this study. Corresponding wind tunnel data is available for all cases. For each booster nose shape, solutions were obtained at two Mach numbers (0.9 and 1.1) and two angles of attack $\left(0^{\circ}\right.$ and $\left.4^{\circ}\right)$, at nominal wind tunnel conditions. The surface pressure at 619 locations was saved at each time step (pressure time histories). These locations nominally included all the locations of the wind tunnel model, plus additional circumferential locations to provide a denser distribution. Unfortunately, as these cases were run before model fabrication was complete, some of the wind tunnel locations were not duplicated (locations were moved added, or deleted). In addition to the pressure time histories, the entire surface solution was saved every 20th time step, and at every 200th time step a $Z=0$ cut plane and a Mach $=1$ or Mach $=1.2$ iso-surface was saved (depending on freestream Mach number).

Table 1: CFD case matrix.

\begin{tabular}{clllllllrc} 
Case & Designation & Nose geometry & Mach & $\alpha$ & $\gamma$ & $R e_{D}$ & $T_{\infty}$ & $\mathrm{m}$ & $\mathrm{T}$ \\
\hline 1 & AAT-01-1.0-01 & sharp on-axis & 0.9 & 0 & 1.4 & 2.07 & 481.9 & 590,000 & 1.105 \\
4 & AAT-01-1.0-04 & sharp on-axis & 0.9 & 4 & 1.4 & 2.07 & 481.9 & 55,000 & 0.106 \\
5 & AAT-01-1.0-05 & sharp on-axis & 1.1 & 0 & 1.4 & 2.07 & 481.9 & 45,000 & 0.087 \\
6 & AAT-01-1.0-06 & sharp on-axis & 1.1 & 4 & 1.4 & 2.07 & 481.9 & 45,000 & 0.087 \\
7 & AAT-02-1.0-07 & sharp canted & 0.9 & 0 & 1.4 & 2.07 & 481.9 & 145,000 & 0.279 \\
8 & AAT-02-1.0-08 & sharp canted & 0.9 & 4 & 1.4 & 2.07 & 481.9 & 45,000 & 0.087 \\
9 & AAT-02-1.0-09 & sharp canted & 1.1 & 0 & 1.4 & 2.07 & 481.9 & 45,000 & 0.087 \\
10 & AAT-02-1.0-10 & sharp canted & 1.1 & 4 & 1.4 & 2.07 & 481.9 & 45,000 & 0.087 \\
11 & AAT-03-1.0-11 & blunt on-axis & 0.9 & 0 & 1.4 & 2.07 & 481.9 & 55,000 & 0.106 \\
12 & AAT-03-1.0-12 & blunt on-axis & 0.9 & 4 & 1.4 & 2.07 & 481.9 & 45,000 & 0.087 \\
13 & AAT-03-1.0-13 & blunt on-axis & 1.1 & 0 & 1.4 & 2.07 & 481.9 & 45,000 & 0.087 \\
14 & AAT-03-1.0-14 & blunt on-axis & 1.1 & 4 & 1.4 & 2.07 & 481.9 & 45,000 & 0.087 \\
15 & AAT-04-1.0-15 & blunt canted & 0.9 & 0 & 1.4 & 2.07 & 481.9 & 55,000 & 0.106 \\
16 & AAT-04-1.0-16 & blunt canted & 0.9 & 4 & 1.4 & 2.07 & 481.9 & 45,000 & 0.087 \\
17 & AAT-04-1.0-17 & blunt canted & 1.1 & 0 & 1.4 & 2.07 & 481.9 & 45,000 & 0.087 \\
18 & AAT-04-1.0-18 & blunt canted & 1.1 & 4 & 1.4 & 2.07 & 481.9 & 55,000 & 0.106 \\
19 & AAT-05-1.0-19 & ogive on-axis & 0.9 & 0 & 1.4 & 2.07 & 481.9 & 55,000 & 0.106 \\
20 & AAT-05-1.0-20 & ogive on-axis & 0.9 & 4 & 1.4 & 2.07 & 481.9 & 45,000 & 0.087 \\
21 & AAT-05-1.0-21 & ogive on-axis & 1.1 & 0 & 1.4 & 2.07 & 481.9 & 45,000 & 0.087 \\
22 & AAT-05-1.0-22 & ogive on-axis & 1.1 & 4 & 1.4 & 2.07 & 481.9 & 55,000 & 0.106 \\
40 & AAT-17-1.0-40 & canted ogive & 0.9 & 0 & 1.4 & 2.07 & 481.9 & 135,000 & 0.260 \\
42 & AAT-17-1.0-42 & canted ogive & 0.9 & 0 & 1.4 & 2.07 & 481.9 & 95,000 & 0.183 \\
43 & AAT-17-1.0-33 & canted ogive & 0.9 & 0 & 1.4 & 2.07 & 481.9 & 60,000 & 0.115
\end{tabular}

\section{Wind Tunnel Test}

Experimental data were acquired as part of an SLS wind tunnel test that was conducted in the transonic leg of the NASA Ames Unitary Plan Wind Tunnel (11-Foot Transonic Unitary Plan Facility). ${ }^{16,17}$ The primary purpose of that test was to acquire data to define the aeroacoustic environment about the SLS. Two additional days of testing were conducted to collect data with the various nose cones. The test article for these runs was a $2.50 \%$ scale model of the SLS 27000 configuration. Approximately 235 dynamic pressure gages were installed in the model, 169 of which corresponded to locations in the CFD database. Most of the gages that did not match CFD locations were either symmetrically located on the left side of the model from locations that were matched, or purposely placed near protuberances, which were omitted from the 
CFD model. The gage responses were converted to differential pressure, and saved as pressure time histories. Freestream flow conditions were sampled at a lower rate and an average value was used for the entire data record when converting to pressure coefficient. Figure 4 shows the model installed in the 11-ft test section.

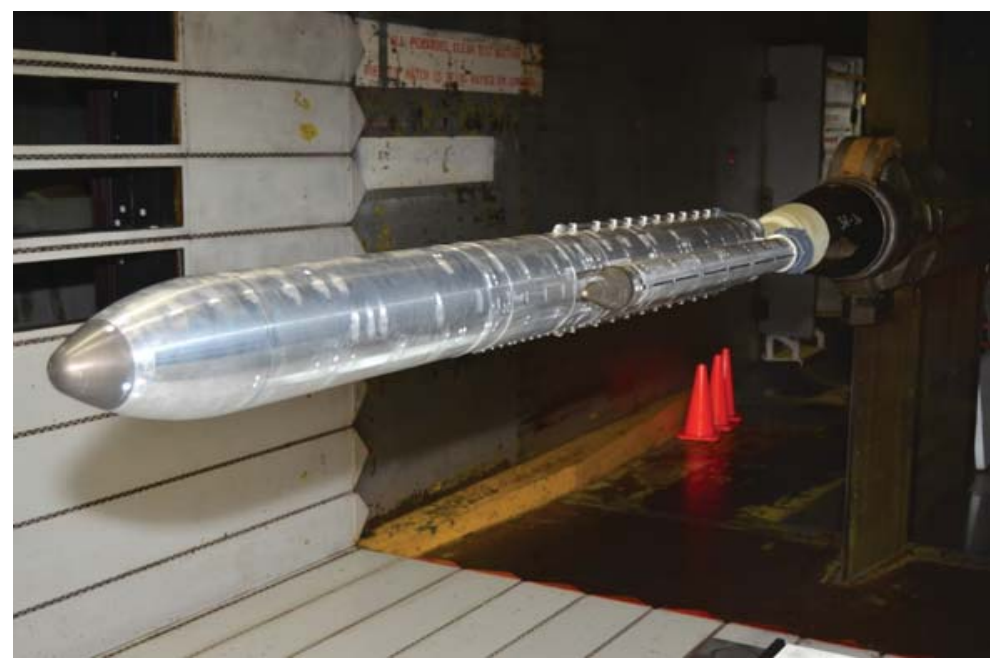

Figure 4: Photograph of SLS configuration installed in the Ames 11-Foot test section.

\section{Results and Discussion}

The results will be discussed in the following sections. The basic flow field will be described using computational results for the baseline case (case $1, \mathrm{M}=0.9, \alpha=0^{\circ}$ ), and at a higher Mach number (case 5 , $\left.\mathrm{M}=1.1, \alpha=0^{\circ}\right)$. Comparisons of computational and experimental results for several booster nose shapes will follow, in both the time domain (in terms of $C_{p, r m s}$ ) and the frequency domain (power spectral density estimates). Finally, an assessment of all the alternate noses in terms of an area-weighted average power metric will be given. This metric-driven assessment highlights one example of how CFD can be used to screen multiple configurations for buffet environments. As part of this analysis, a method to analyze the short time durations typical of these CFD calculations will be described.

\section{VI.A. Basic flowfield}

The Mach 0.9, $0^{\circ}$ angle-of-attack condition (case 1) was known from a previous SLS wind tunnel test to have elevated unsteady pressure levels around the forward attach point, and thus was chosen as the baseline condition for this study. The basic flow field in this forward attach region at this condition consists of an oscillating shock/separation at the booster nose cone/cylinder junction, interacting with a wake shed from the forward attach hardware. The cone-cylinder unsteady flow field has been studied in the past, ${ }^{18}$ but the presence of a second body (the core), and especially the attach hardware, further complicates the flow field. Various aspects of the flowfield are shown in Figs. 5-8. Surface pressure and the near-surface Mach number are shown in Fig. 5 for two different times. The view is of the core, looking in from the booster, along the Y-axis. Visible in the images are two curved lines, which are the forward and aft inter-tank flanges. The increase in Mach number and corresponding decrease in pressure ahead of the attach hardware is due to the expansion of the flow around the booster cone/cylinder junction. The flow decelerates through the cone/cylinder shock. A large separated region is created behind the forward attach hardware. In the wake of the attach hardware, one can see a low pressure region moving from one side to another, with the tail of the wake extending downstream on the opposite side. This vortex shedding from the attach hardware can also be seen in the surface streamline plots presented in Fig. 6. Three images are shown encompassing one cycle of the shedding sequence. The low pressure of the vortex core (dark blue region) just forward of the aft inter-tank flange moves from one side of the vehicle to the other. Another view is of sonic iso-surfaces, given in Fig. 7. Again three instances in time are shown. The movement of the cone/cylinder shock is hard to see in these still images, but the wake shedding aspect is apparent.

The resultant pressure fluctuations are seen in Fig. 8a-b, contour plots of the root-mean-square of the 
pressure fluctuations $\left(C_{p, r m s}\right)$. The core stage ahead of the booster nose is very quiet, with low values of $C_{p, r m s}$. There is a region of increased pressure fluctuations behind the forward attach hardware, with a 'hot spot' on the centerline between core and booster. The fluctuations decrease moving away from this region, both longitudinally and circumferentially, until the aft attach region is reached, where the fluctuation levels increase once again, but not to the same levels as in the forward attach region. An area of increased fluctuation wraps around the booster just aft of the cone/cylinder junction, due to the oscillating shock.

At a Mach number of 1.1, the booster cone/cylinder shock becomes steady, and the pressure fluctuations on the side of the booster are gone. The fluctuations in the attach region between the core and booster diminish, but are still present, as seen in Fig. 8c-d. Unsteady flow is seen on the core forward of the booster for this flow condition.

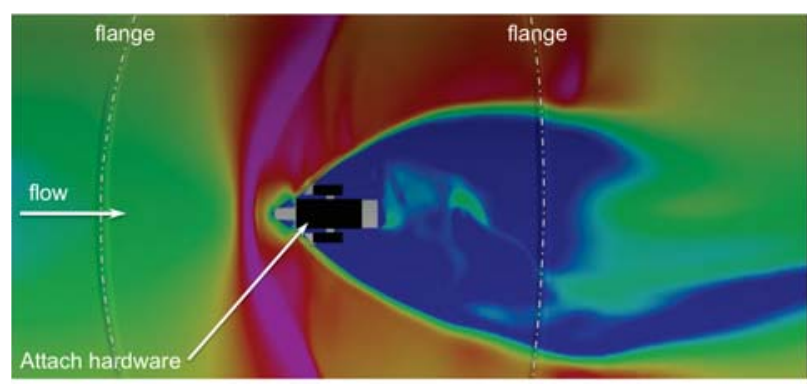

(a) Mach number, time 1

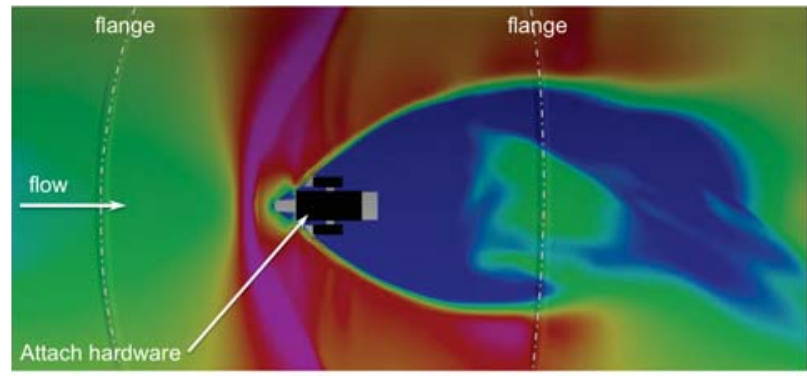

(c) Mach number, time 2

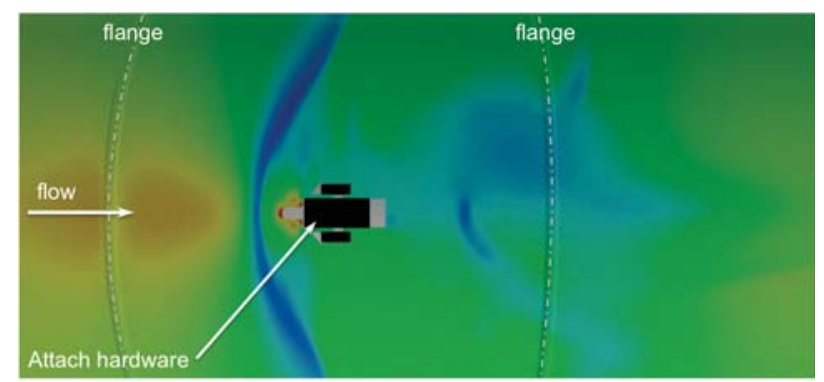

(b) Pressure, time 1

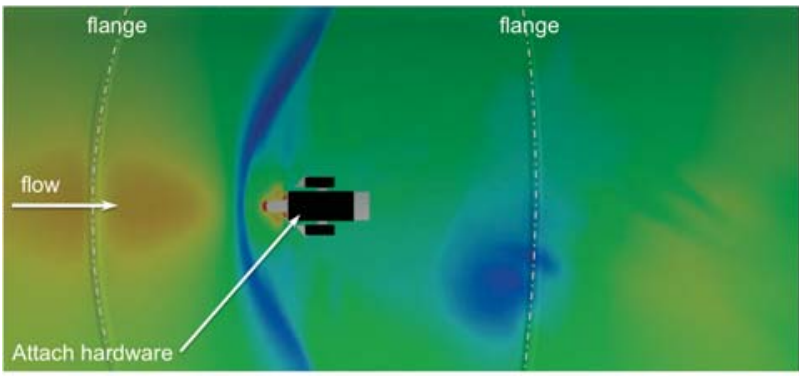

(d) Pressure, time 2

Figure 5: Computed near-surface Mach number and surface pressure $\left(\mathrm{M}=0.9, \alpha=0^{\circ}\right)$.

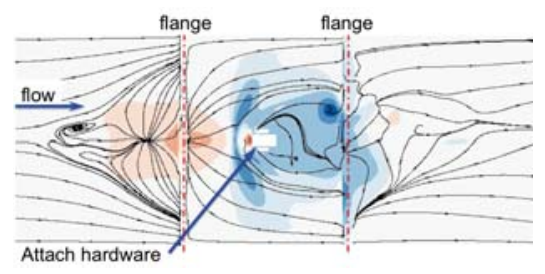

(a) time 1

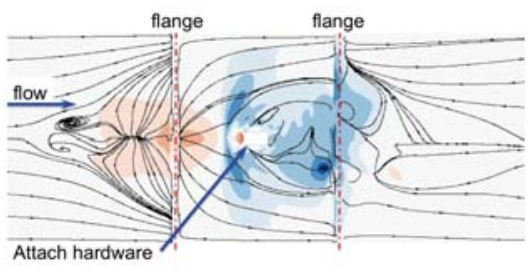

(b) time 2

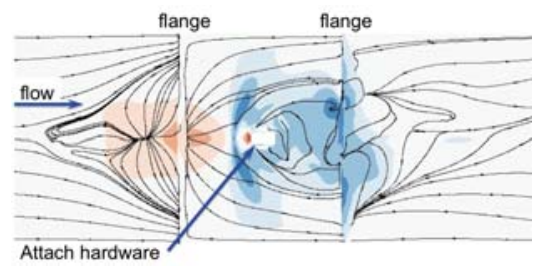

(c) time 3

Figure 6: Computed surface streamlines for three time steps $\left(\mathrm{M}=0.9, \alpha=0^{\circ}\right)$.

\section{VI.B. Comparisons with wind tunnel data}

A comparison between computed and measured wind tunnel data, in terms of $C_{p, r m s}$ contours for the core and booster, is given in Fig. 9 for case 1 (Mach 0.9, $\alpha=0^{\circ}$ ). On the left is the computational prediction, and on the right is the corresponding wind tunnel result. The images are of the core and the booster lower surface facing the core. The location of the elevated $C_{p, r m s}$ levels are generally matched, although the peak magnitude is lower for the CFD prediction than the wind tunnel data. The CFD predicts slightly higher $C_{p, r m s}$ levels in the aft attach region. The pattern of $C_{p, r m s}$ contours on the core are essentially mirrored on the booster. A more quantitative comparison is given in Fig. 10. The left side of this figure contains comparisons for the core, and the right side contains results for the booster. Each side is further subdivided 


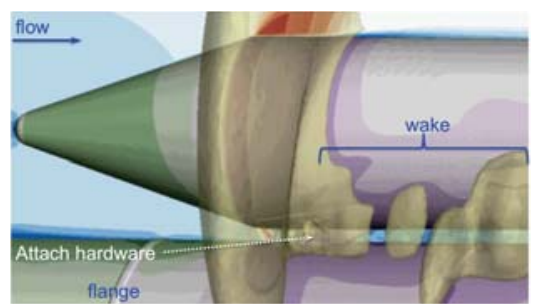

(a) time 1

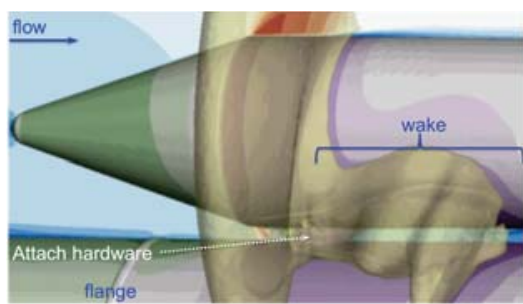

(b) time 2

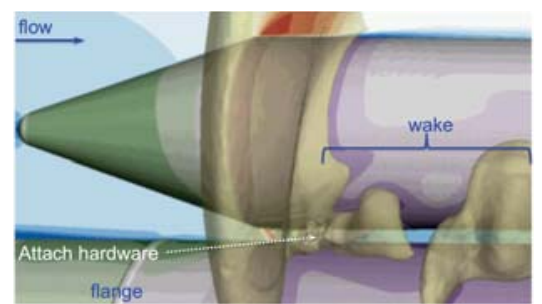

(c) time 3

Figure 7: Sonic iso-surfaces for three time steps $\left(\mathrm{M}=0.9, \alpha=0^{\circ}\right)$.
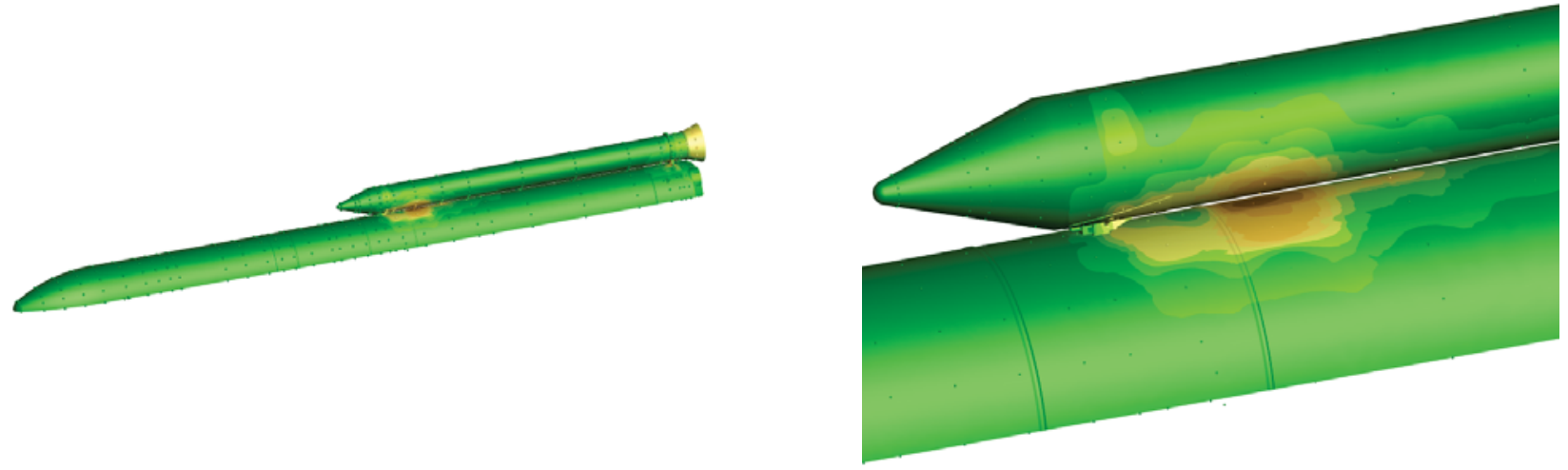

(a) overall view, $M=0.9, \alpha=0^{\circ}$

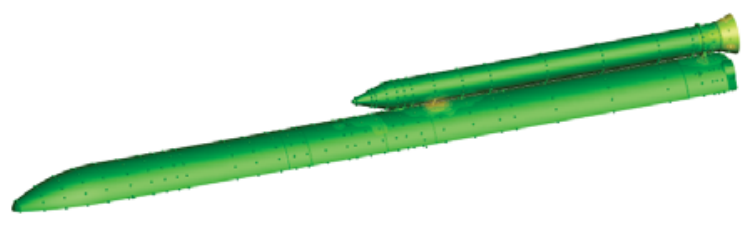

(c) overall view, $M=1.1, \alpha=0^{\circ}$ (b) forward attach region, $M=0.9, \alpha=0^{\circ}$

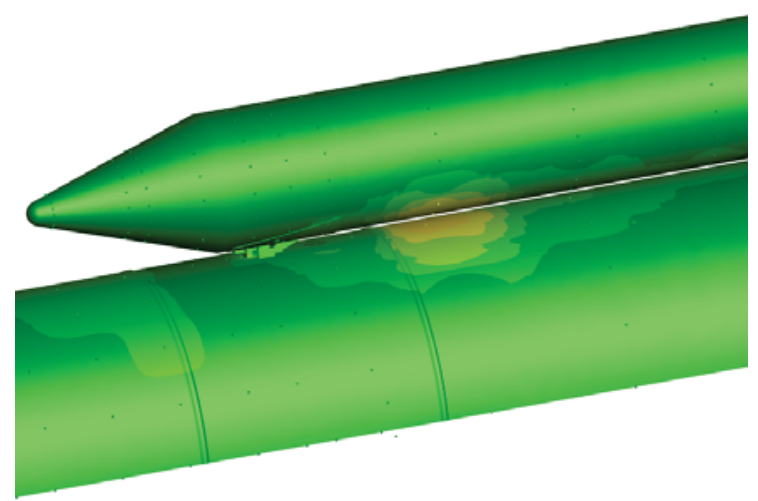

(d) forward attach region, $M=1.1, \alpha=0^{\circ}$

Figure 8: Computed $C_{p, r m s}$ contours.

by azimuth angle. The elevated $C_{p, r m s}$ levels behind the forward attach point and in the aft attach region are clearly seen.

Comparisons in the frequency domain, in terms of the power spectral density (PSD), will be shown at 4 representative locations, 3 on the vehicle core, and one on the booster. The 4 locations are shown in Fig. 11 to orient the reader. Figure 12a shows a comparison for an off-centerline location behind the forward attach hardware $\left(\mathrm{X} / \mathrm{L}=0.59, \phi=247.5^{\circ}\right)$. There is a dominant frequency (large peak in the PSD power) associated with the oscillation of the wake behind the forward attach hardware. The predicted frequency of this peak is lower than that observed in the wind tunnel by about $25 \%$, while the magnitude of this peak is over-predicted by a factor of nearly 7 . At frequencies less than the peak, the CFD over-predicts the magnitudes by a factor of roughly 3 . Figure. $12 \mathrm{~b}$ shows a comparison at the same longitudinal station, but on-centerline $(\mathrm{X} / \mathrm{L}=0.59$, $\phi=270^{\circ}$ ). The peak frequency is double the off-centerline value, which is consistent with a vortex shedding phenomena wherein a low-pressure vortex core oscillates back and forth across the centerline, passing the 
centerline twice for each cycle. The predicted dominant frequency is again lower, by $20 \%$, but at this location the CFD under-predicts the wind tunnel magnitude by $35 \%$. At the lower frequencies the CFD over-predicts in some instances, and in other instances under-predicts, the wind tunnel magnitudes. Figure 12c presents a comparison for the same longitudinal station, but on the booster, while Fig. 12d shows the results for a core location in the aft attach region. In all cases, the frequency response appears shifted, with the predicted frequencies lower than the wind tunnel results. Additional CFD cases, which examined grid resolution and time step size, have improved but not completely resolved the discrepancy in frequency. ${ }^{8}$ The magnitude of the power estimates are over-predicted in some cases, under-predicted in others. The reason or reasons for these discrepancies are not understood at this time.

The $C_{p, r m s}$ and PSD comparisons for the same locations for case 5 (Mach 1.1, $\alpha=0^{\circ}$ ) are given in Figs. 13 and 14. The $C_{p, r m s}$ magnitudes (Fig. 13) are reduced in the forward attach region compared to the $\mathrm{M}=0.9$ baseline case, but nearly the same in the aft attach region. The frequencies are shifted higher (Fig. 14), by more than a factor of two. The previous $\mathrm{M}=0.9$ data is shown as grey lines to provide a point of reference. The CFD again consistently under-predicts the peak frequency, but in this case the agreement is better, with the CFD low by $15 \%$. The magnitudes of the peak frequency are in better agreement as well.

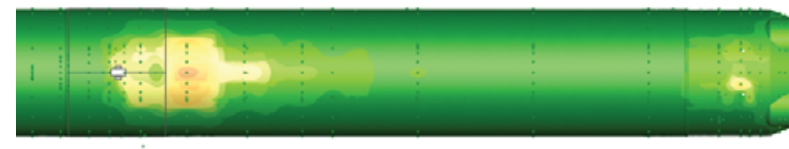

(a) core: computed

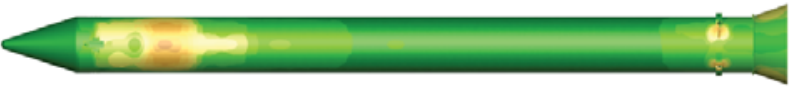

(c) booster: computed

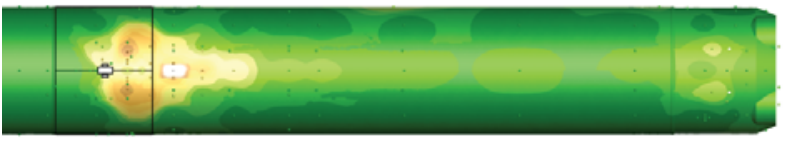

(b) core: wind tunnel

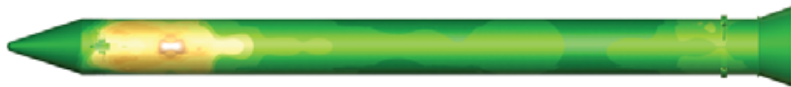

(d) booster: wind tunnel

Figure 9: Comparison of computed and measured $C_{p, r m s}$ contours for the baseline nose (case $1, M=0.9, \alpha=0^{\circ}$ ). 


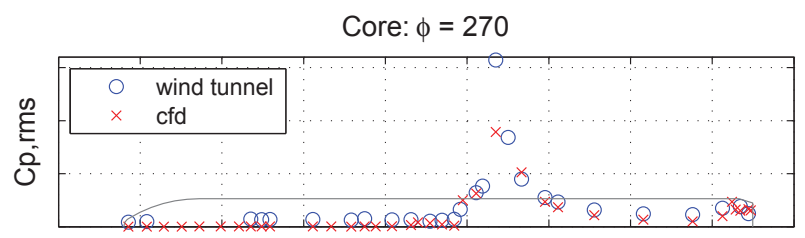

Core: $\phi=247,293$

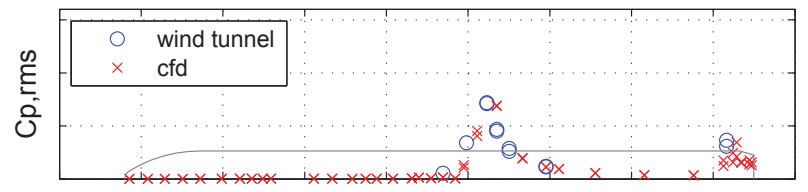

Core: $\phi=225,315$

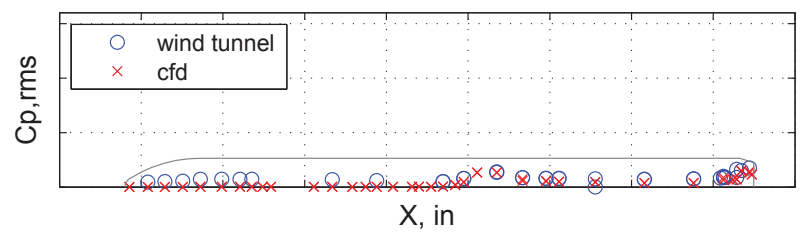

Booster: $\phi=75,90,105$

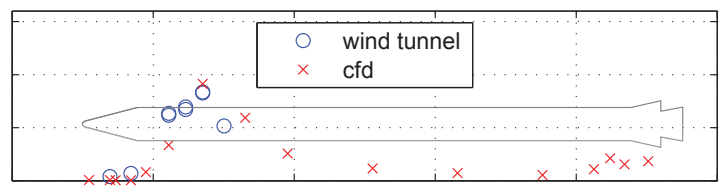

Booster: $\phi=67$ (60), 113 (120)

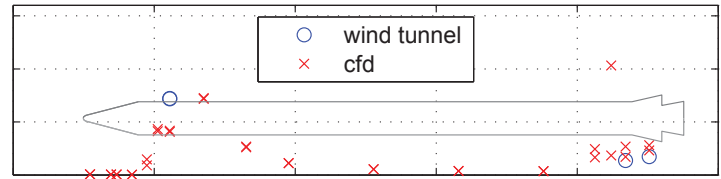

Booster: $\phi=45,135$

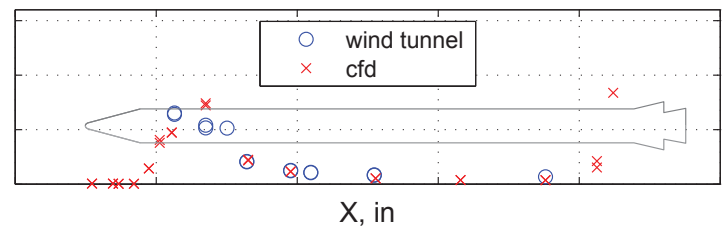

Figure 10: Comparison of computed and measured $C_{p, r m s}$ values for the baseline nose (case $1, M=0.9, \alpha=0^{\circ}$ ).

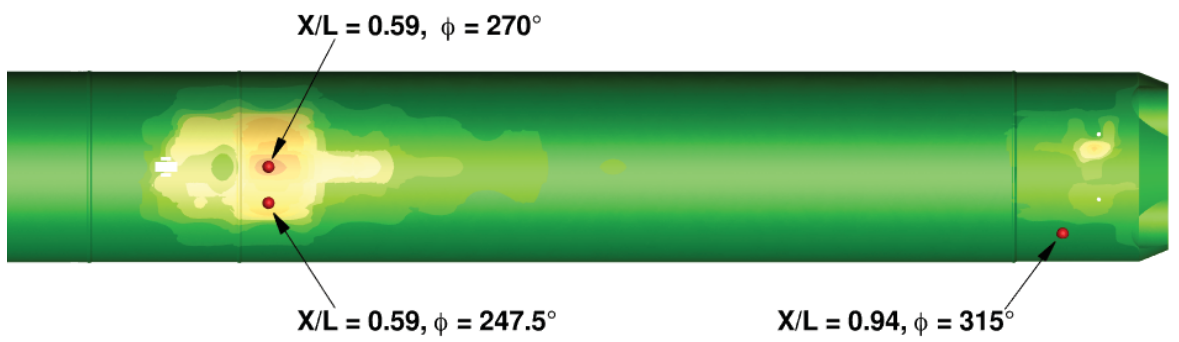

(a) Locations for PSD comparisons, core

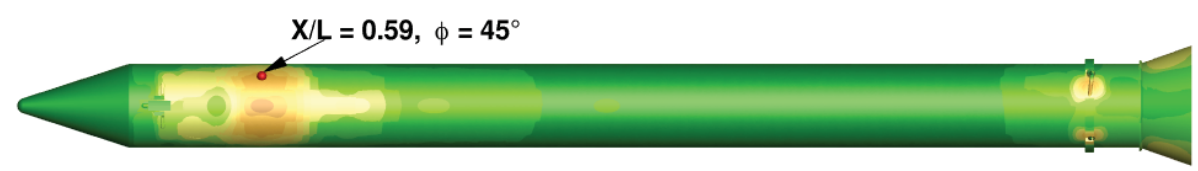

(b) Locations for PSD comparisons, booster

Figure 11: Locations for power spectral density comparisons. 


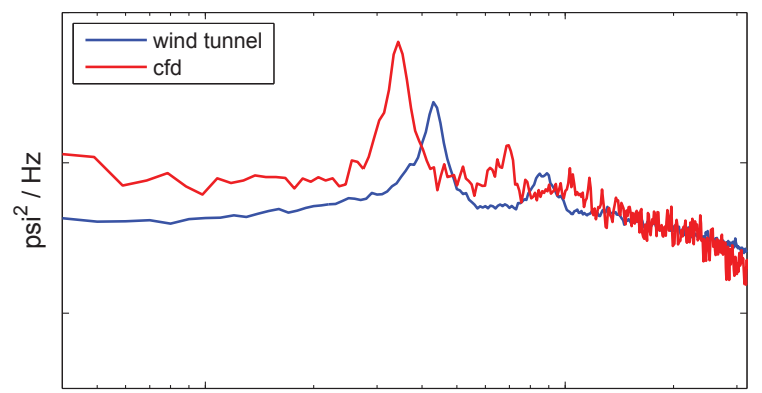

$\mathrm{f}, \mathrm{Hz}$ (model scale)

(a) forward attach region, core, $\mathrm{X} / \mathbf{L}=\mathbf{0 . 5 9 1}, \phi=247.5^{\circ}$

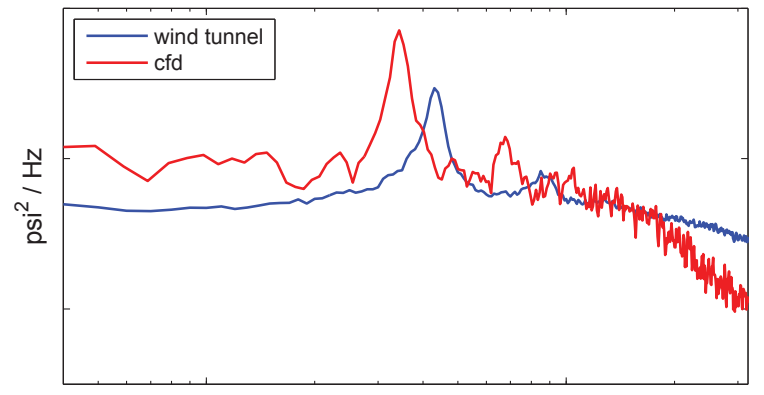

f, $\mathrm{Hz}$ (model scale)

(c) forward attach region, booster, $\mathbf{X} / \mathbf{L}=\mathbf{0 . 5 9 1}, \phi=45.0^{\circ}$

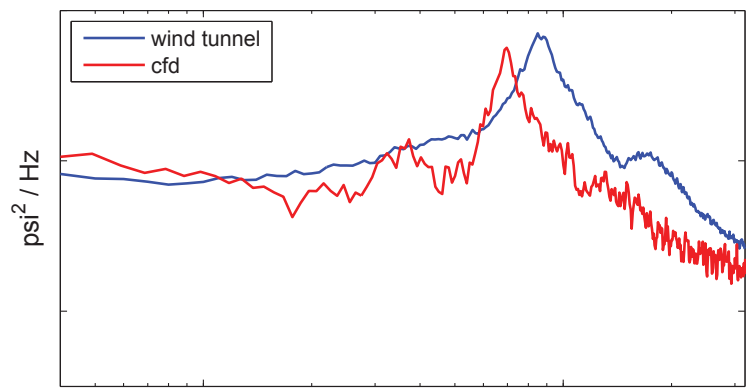

$\mathrm{f}, \mathrm{Hz}$ (model scale)

(b) forward attach region, core, $\mathbf{X} / \mathbf{L}=\mathbf{0 . 5 9 1}, \phi=270.0^{\circ}$

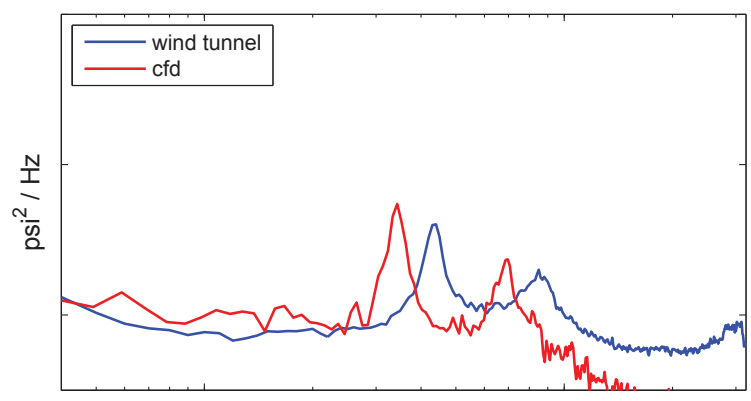

f, $\mathrm{Hz}$ (model scale)

(d) aft attach region, core, $\mathbf{X} / \mathbf{L}=\mathbf{0 . 9 8 0}, \phi=315.0^{\circ}$

Figure 12: Comparison of power spectral densities for the baseline nose (case $1, M=0.9, \alpha=0^{\circ}$ ).
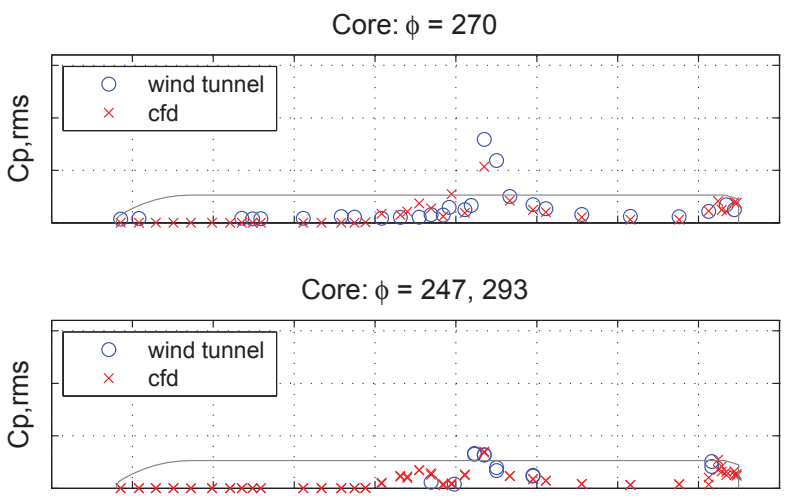

Core: $\phi=225,315$

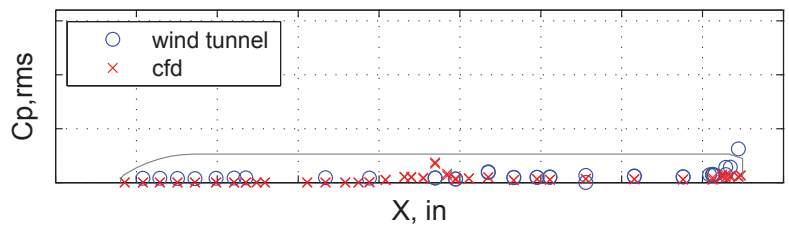

Booster: $\phi=75,90,105$

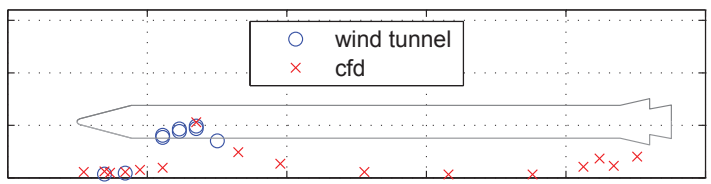

Booster: $\phi=67(60), 113(120)$

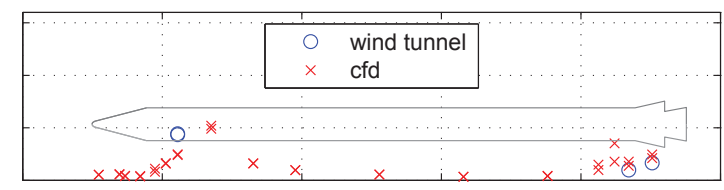

Booster: $\phi=45,135$

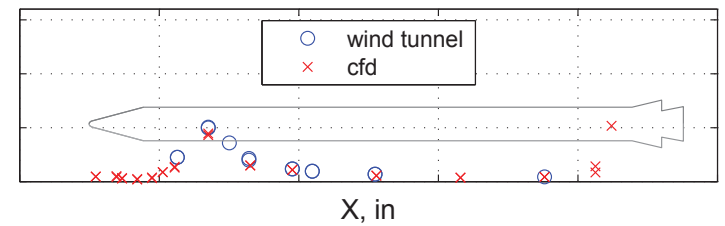

Figure 13: Comparison of computed and measured $C_{p, r m s}$ values for the baseline nose (case $5, M=1.1, \alpha=0^{\circ}$ ). 


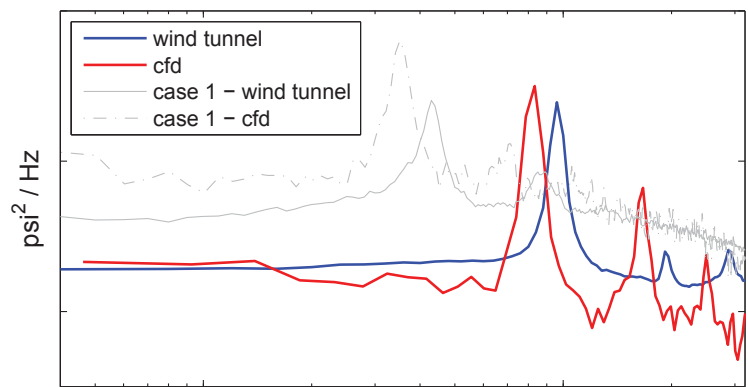

$\mathrm{f}, \mathrm{Hz}$ (model scale)

(a) forward attach region, core, $\mathrm{X} / \mathrm{L}=\mathbf{0 . 5 9 1}, \phi=247.5^{\circ}$

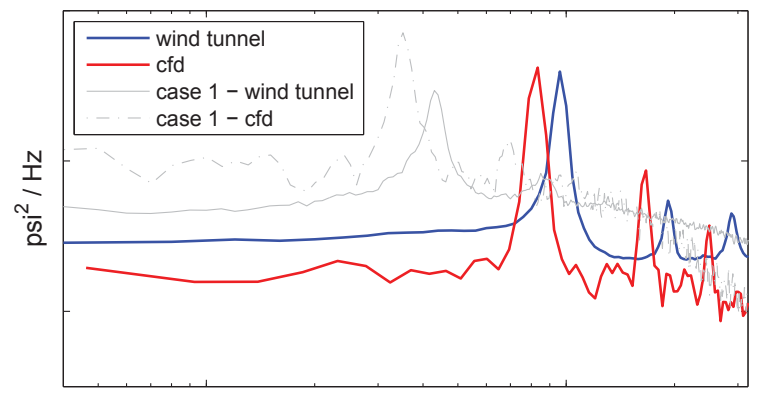

f, $\mathrm{Hz}$ (model scale)

(c) forward attach region, booster, $\mathrm{X} / \mathrm{L}=\mathbf{0 . 5 9 1}, \phi=45.0^{\circ}$

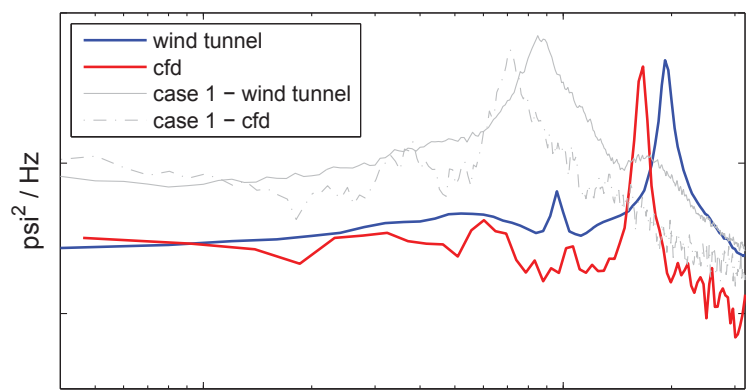

f, $\mathrm{Hz}$ (model scale)

(b) forward attach region, core, $\mathrm{X} / \mathbf{L}=\mathbf{0 . 5 9 1}, \phi=270.0^{\circ}$

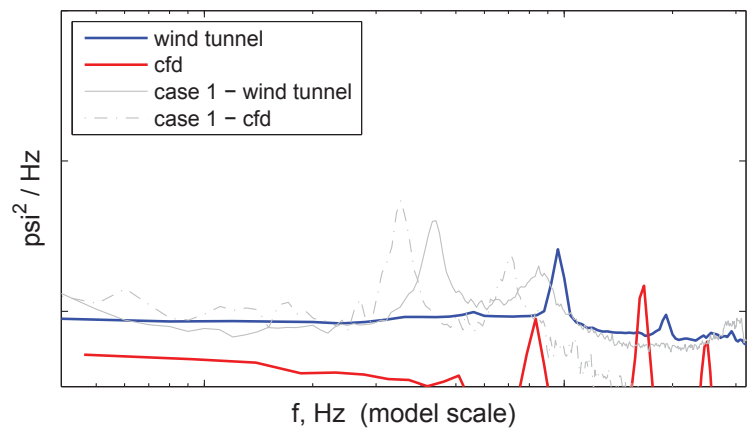

(d) aft attach region, core, $\mathbf{X} / \mathbf{L}=\mathbf{0 . 9 8 0}, \phi=315.0^{\circ}$

Figure 14: Comparison of power spectral densities for the baseline nose (case $5, M=1.1, \alpha=0^{\circ}$ ). 


\section{VI.C. Effect of canting noses inward}

The effect of canting the baseline nose is shown in Figs. 15- 18. The $C_{p, r m s}$ levels are decreased significantly (by a factor of 1.5-3) in the forward attach region, both on the core and the booster. Canting the nose inward towards the core reduces or eliminates the cone/cylinder shock oscillation and its interaction with the wake. The oscillation is still present on the leeside of the booster, and in fact is worse due to the increased angle between cone and cylinder, resulting in (higher $C_{p, r m s}$ levels) as shown in Fig. 17b. There is little difference between the noses in terms of $C_{p, r m s}$ in the aft attach region. In the frequency domain (Fig. 18), canting the baseline nose lowers the magnitudes and shifts peak frequencies to higher values. The overall agreement between wind tunnel and CFD is much better than for the baseline case, as the power levels are in better agreement, and the frequencies are better aligned (within 10\% in this case). The results for the canted ogive shape are shown in Figs. 19- 22. As with the sharp canted nose, the canted ogive shape significantly decreases the $C_{p, r m s}$ levels in the forward attach region, both on the core and the booster, compared to the baseline nose. The increased $C_{p, r m s}$ levels on the leeside of the booster that were present on the sharp canted nose have been eliminated for the canted ogive, due to removing the sharp angle at the nose/cylinder junction, as seen in Fig. 21b. The frequency shift (Fig. 22), compared to the baseline, is greater than that observed for the sharp canted nose. Overall agreement between CFD and wind tunnel, in terms of peak frequencies, is good (4-7\%), but the power levels in the lower frequencies are overpredicted by the CFD for the core centerline location.

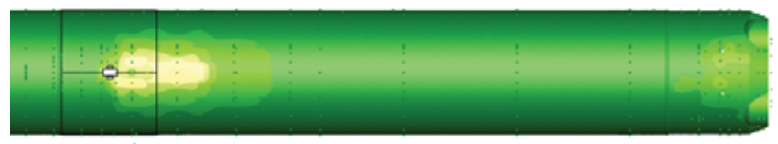

(a) core: computed

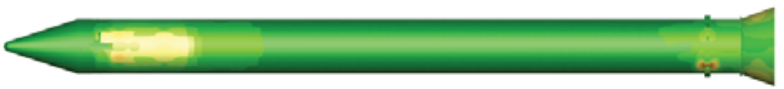

(c) booster: computed

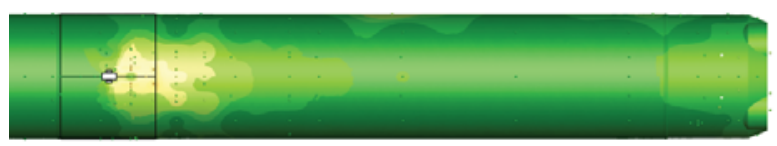

(b) core: wind tunnel

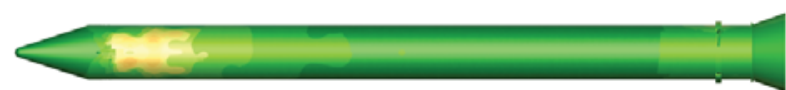

(d) booster: wind tunnel

Figure 15: Comparison of computed and measured $C_{p, r m s}$ contours for the sharp canted nose (case $\left.7, M=0.9, \alpha=0^{\circ}\right)$. 

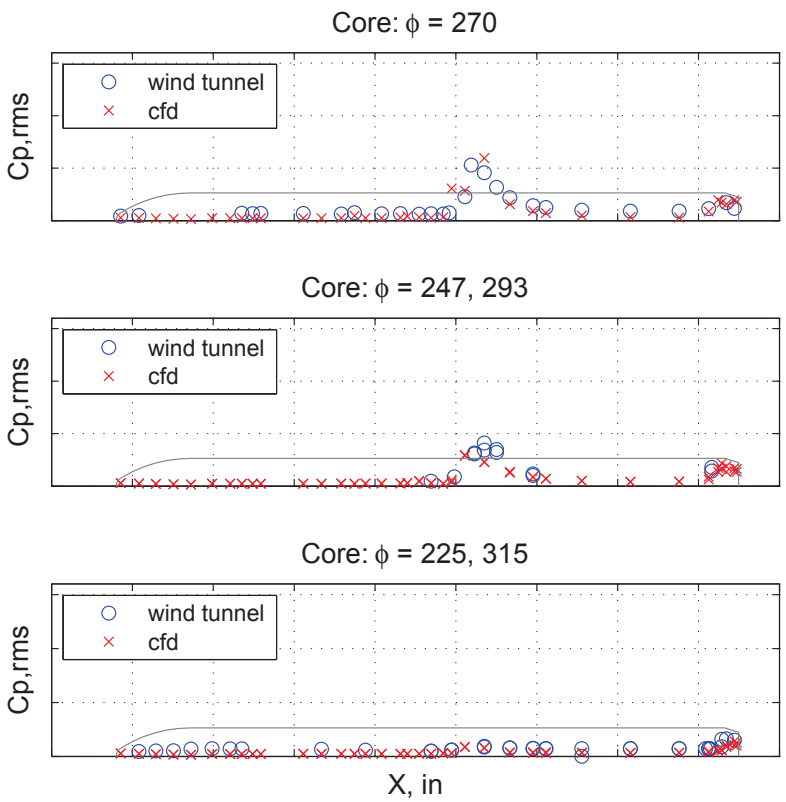
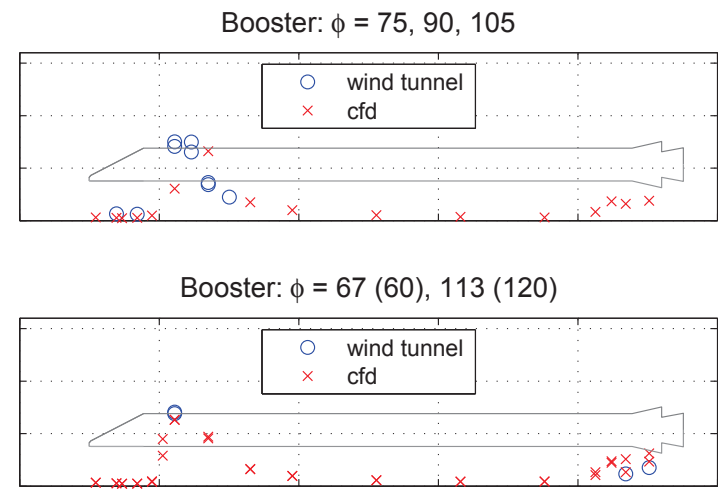

Booster: $\phi=45,135$

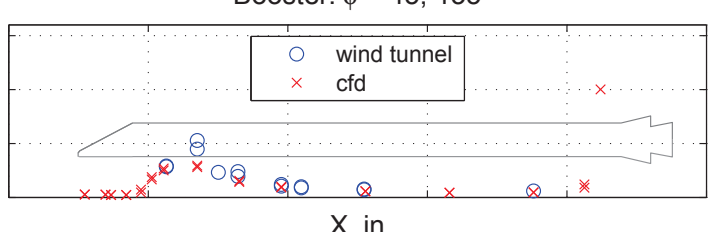

$X$, in

Figure 16: Comparison of computed and measured $C_{p, r m s}$ values for the sharp canted nose (case $\left.7, M=0.9, \alpha=0^{\circ}\right)$.

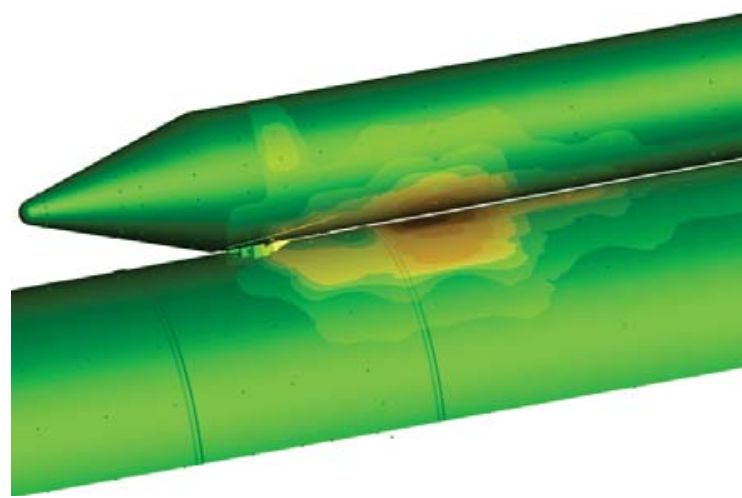

(a) forward attach region, sharp on-axis nose (case 1, baseline)

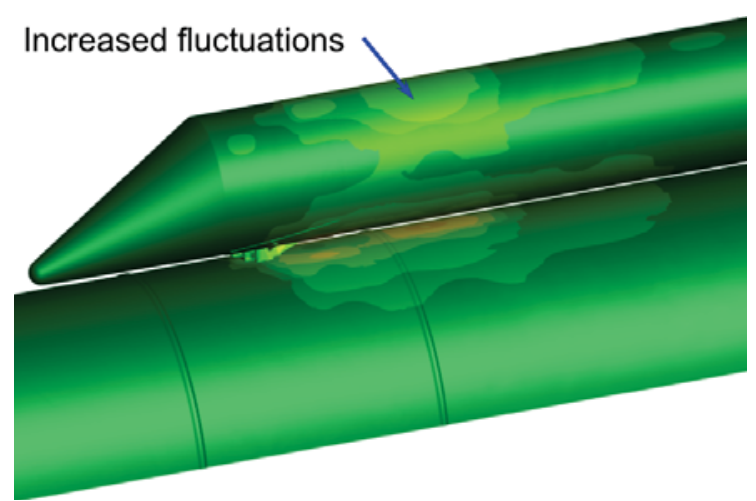

(b) forward attach region, sharp canted nose (case 7)

Figure 17: Computed $C_{p, r m s}$ contours, $M=0.9, \alpha=0^{\circ}$. 


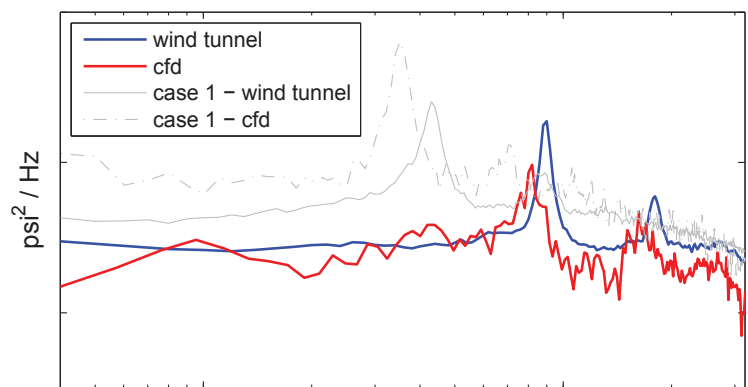

$\mathrm{f}, \mathrm{Hz}$ (model scale)

(a) forward attach region, core, $\mathrm{X} / \mathrm{L}=\mathbf{0 . 5 9 1}, \phi=292.5^{\circ}$

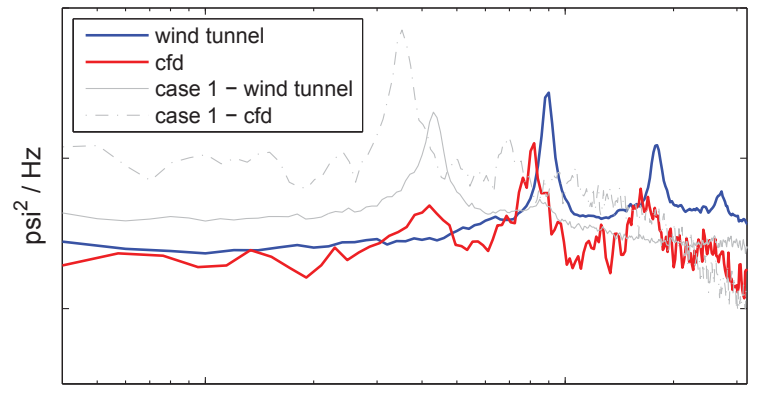

$\mathrm{f}, \mathrm{Hz}$ (model scale)

(c) forward attach region, booster, $\mathrm{X} / \mathrm{L}=\mathbf{0 . 5 9 1}, \phi=45.0^{\circ}$

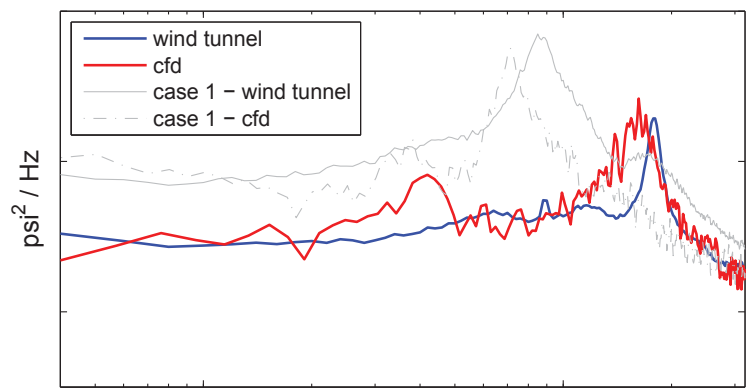

f, $\mathrm{Hz}$ (model scale)

(b) forward attach region, core, $\mathrm{X} / \mathbf{L}=\mathbf{0 . 5 9 1}, \phi=270.0^{\circ}$

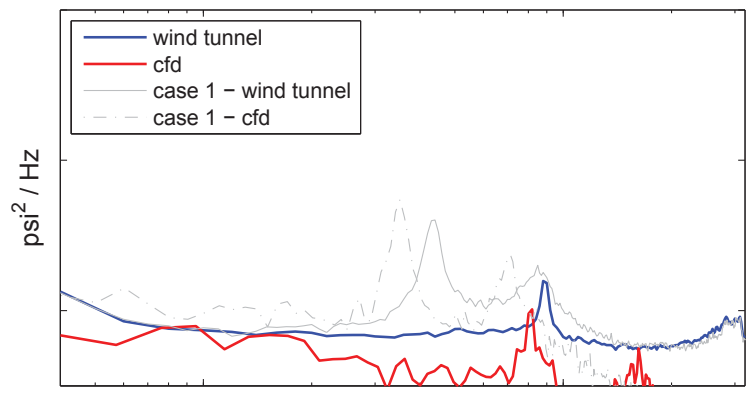

$\mathrm{f}, \mathrm{Hz}$ (model scale)

(d) aft attach region, core, $\mathbf{X} / \mathbf{L}=\mathbf{0 . 9 8 0}, \phi=292.5^{\circ}$

Figure 18: Comparison of power spectral densities for the sharp canted nose (case $\left.7, M=0.9, \alpha=0^{\circ}\right)$.

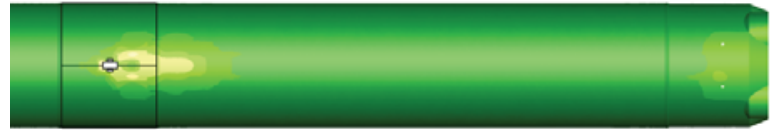

(a) core: computed

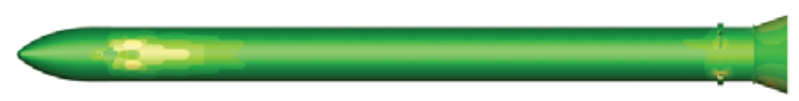

(c) booster: computed

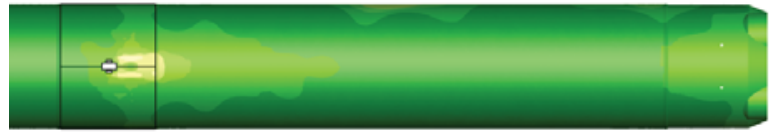

(b) core: wind tunnel

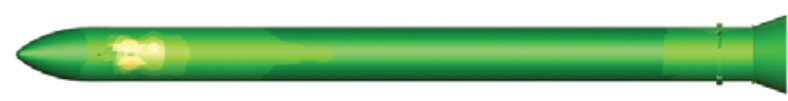

(d) booster: wind tunnel

Figure 19: Comparison of computed and measured $C_{p, r m s}$ contours for the canted ogive nose (case $40, M=0.9, \alpha=0^{\circ}$ ). 

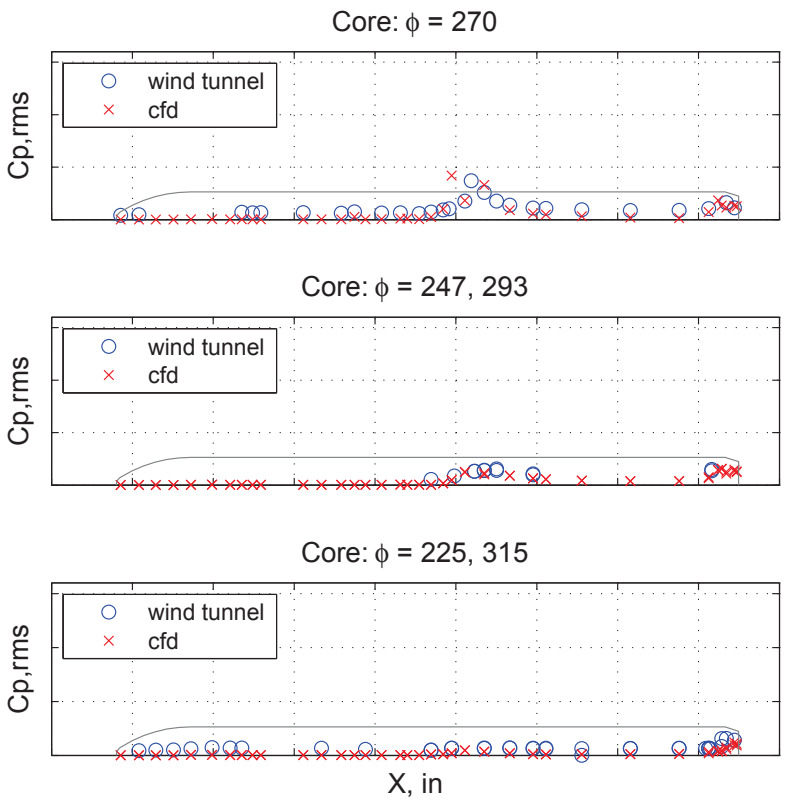
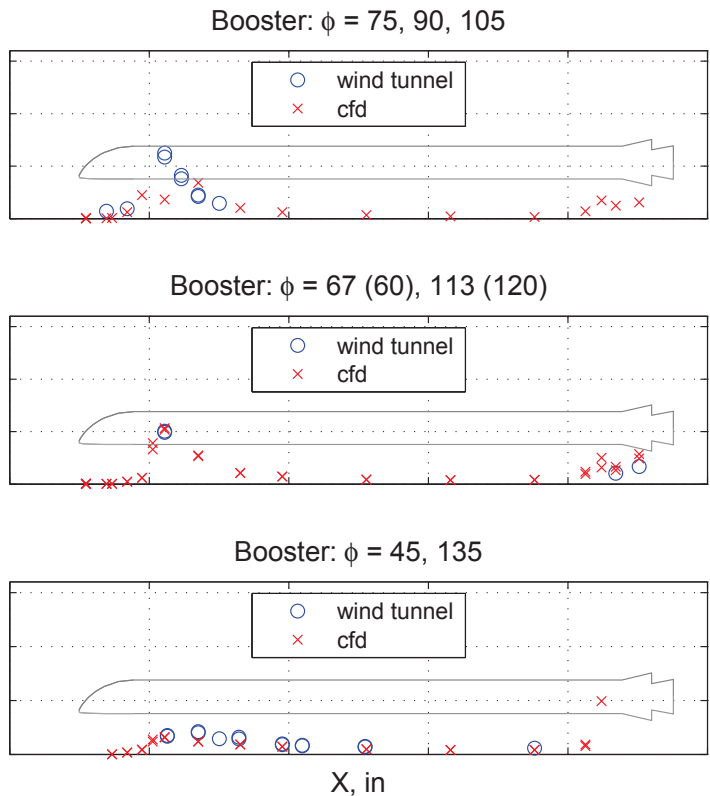

Figure 20: Comparison of computed and measured $C_{p, r m s}$ values for the canted ogive nose (case $40, M=0.9, \alpha=0^{\circ}$ ).

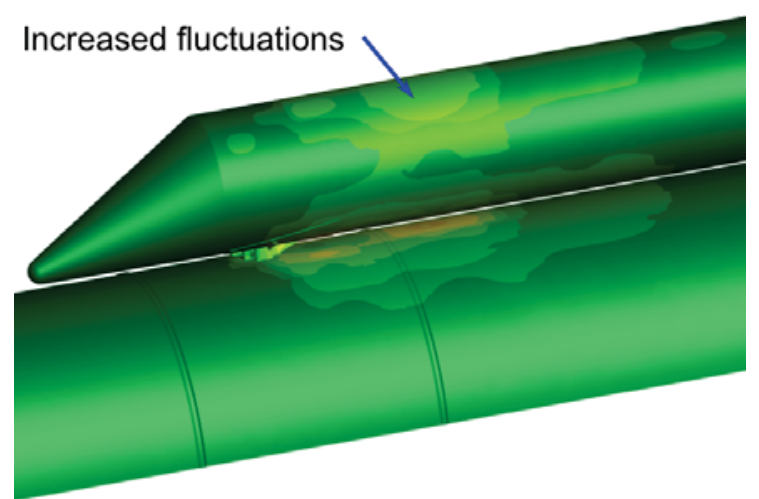

(a) forward attach region, sharp canted nose (case 7)

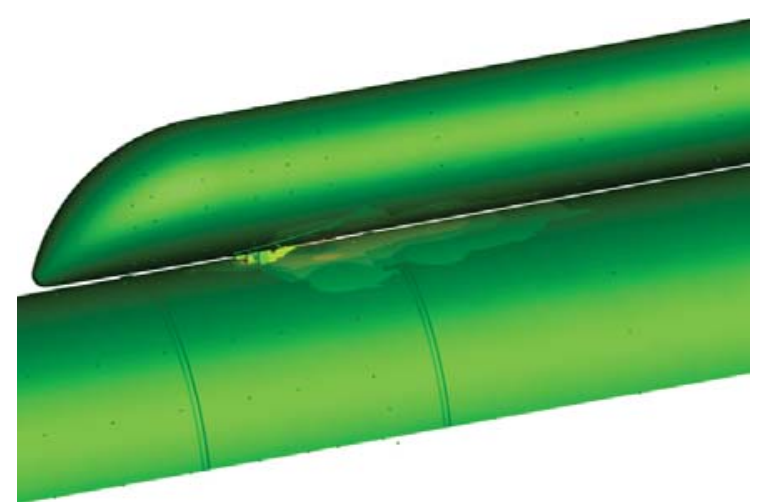

(b) forward attach region,canted ogive nose (case 40)

Figure 21: Computed $C_{p, r m s}$ contours, $M=0.9, \alpha=0^{\circ}$. 


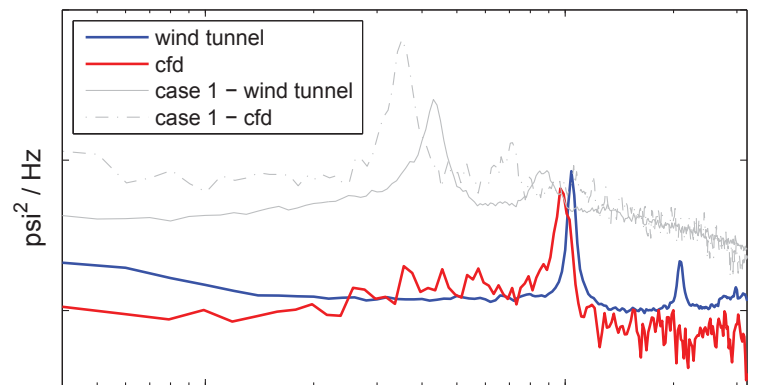

$\mathrm{f}, \mathrm{Hz}$ (model scale)

(a) forward attach region, core, $\mathrm{X} / \mathbf{L}=\mathbf{0 . 5 9 1}, \phi=292.5^{\circ}$

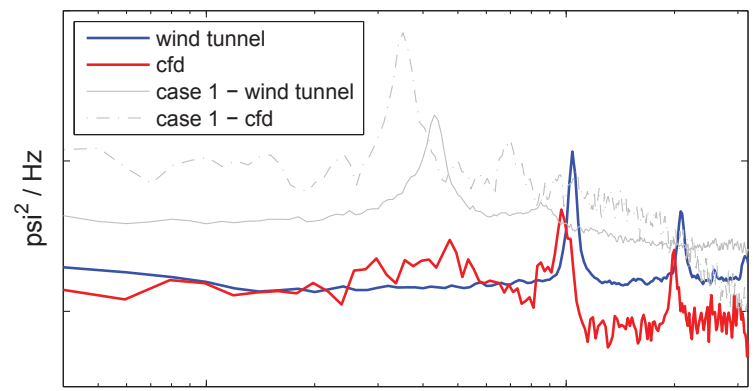

f, $\mathrm{Hz}$ (model scale)

(c) forward attach region, booster, $\mathrm{X} / \mathrm{L}=\mathbf{0 . 5 9 1}, \phi=45.0^{\circ}$

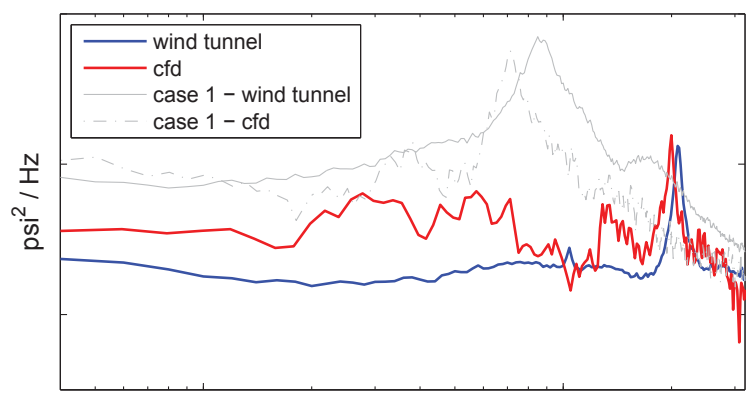

f, $\mathrm{Hz}$ (model scale)

(b) forward attach region, core, $\mathrm{X} / \mathbf{L}=\mathbf{0 . 5 9 1}, \phi=270.0^{\circ}$

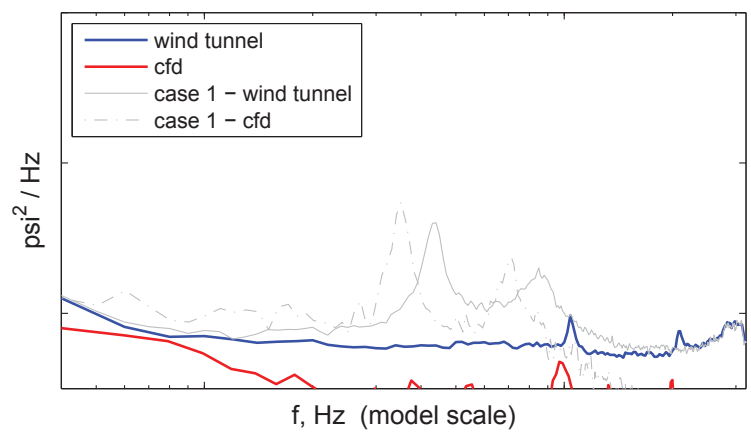

(d) aft attach region, core, $\mathbf{X} / \mathbf{L}=\mathbf{0 . 9 8 0}, \phi=292.5^{\circ}$

Figure 22: Comparison of power spectral densities for the canted ogive nose (case $\left.40, M=0.9, \alpha=0^{\circ}\right)$. 


\section{VI.D. Low frequency resolution and wavelet analysis}

The power spectral density estimates in the previous section were computed using a periodogram averaging method (Welch's method ${ }^{19}$ ), which divides the data record into overlapping blocks, computes the periodogram for each block using a Fast Fourier Transform (FFT), and averages the results. The purpose is to reduce the variance in the estimated power at a given frequency. The reduction in variance comes at the expense of frequency resolution, since frequency resolution is inversely proportional to the number of points in a block. More blocks means more averages, but fewer points in each block and thus larger frequency bins. The effect of the number of blocks is demonstrated in Fig. 23a. Here a data record of 55,000 samples is used, corresponding to a typical short-duration CFD case. The curves represent 1, 5, 14, and 22 overlapping blocks $(\mathrm{Nb})$. The previous short duration PSDs (cases 4 and 5) were computed using 14 blocks. The single-block PSD is very spiky in nature. Averaging smooths out the distribution, but at the cost of decreasing frequency resolution (larger frequency bin sizes). Note how the first point is shifted to the right. All power in the signal below this value is represented in the first frequency bin.

The peak structural response of the vehicle is not necessarily driven by the frequency of peak power. Several modes respond to lower frequencies, so a means to retain resolution at the lower end of the frequency spectrum was desired. Wavelet analysis with the continuous wavelet transform $(\mathrm{CWT})^{20,21}$ was used to maintain this resolution. Wavelet analysis is a means to examine the signal as a function of both time (often referred to as location) and frequency. It is similar to short time-series Fourier analysis, but with a different basis function; in our case a Morley wavelet. The shape of the wavelet is shown in Fig. 24. The width of the wavelet (the scale) is proportional to the frequency. The wavelet is passed through the signal (shifted repeatedly in time), the product of the wavelet and signal is integrated, and the result used to estimate the power at a given frequency. If a given frequency is present in the signal, the integration will yield a larger value than when it is not. By stretching the wavelet horizontally (changing the scale) different frequencies can be estimated, and thus the power at different frequencies and time can be estimated.

A pseudo-PSD similar to the FFT-based PSD can be generated by integrating the CWT for all times at each frequency. An example of the process is given in Fig. 25. On the left is the power in the signal at various scales as a function of time. On the right is the pseudo-PSD result after integrating. An FFT-generated PSD is shown as well, using 14 blocks such as used in the previous section. There are several obvious differences, such as the peak being broadened for the CWT, while the peak magnitude is reduced. The overall power in the signal, as measured by integrating either curve across all frequencies, is the same. The primary benefit of this wavelet approach is the increased frequency resolution in the lower frequency range. The MatLab ${ }^{\circledR}$ code used to generate the CWT-based PSD is given in the Appendix.

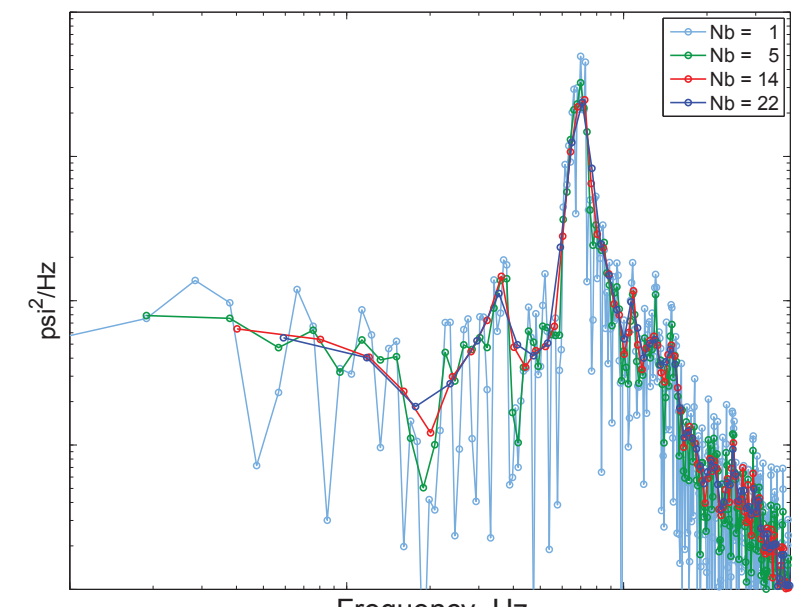

Frequency, $\mathrm{Hz}$

Figure 23: Example of Welch's method showing effect of block averages.

\section{VI.E. Attach region metric and booster nose shape comparisons}

Prior to the wind tunnel test, a metric was devised to rank the noses in terms of area-weighted average power in a region extending from just aft of the forward attach point to just ahead of the aft attach point, extending 


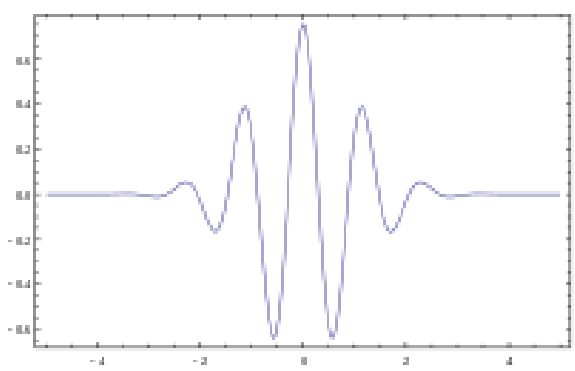

Figure 24: Morley wavelet used in wavelet analysis.

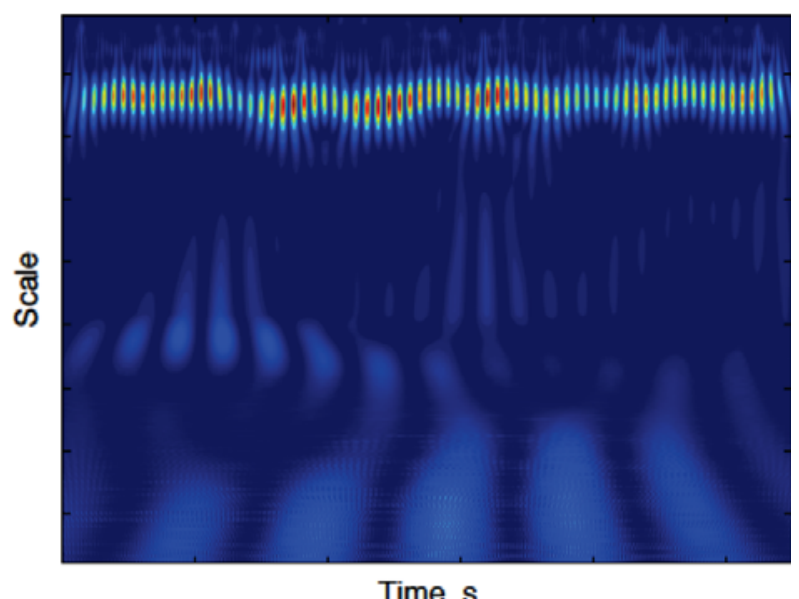

(a) Continuous Wavelet Transform

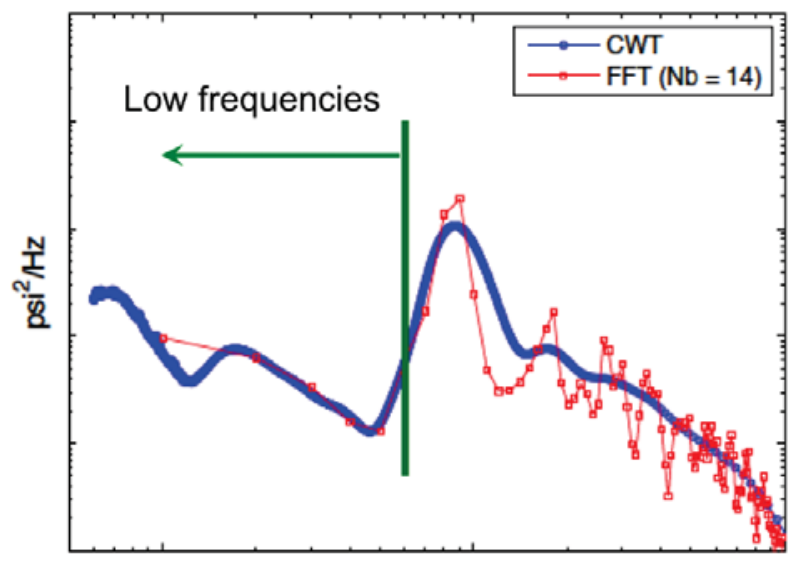

Frequency, $\mathrm{Hz}$

(b) CWT-generated PSD

Figure 25: Example of pseudo-PSD generation using Continuous Wavelet Transform.

$45^{\circ}$ to either side. Reduction in this metric was used as an indicator of likely reduction in buffet loads. The metric was computed using the wavelet-based pseudo-PSD for 45 locations on the core. A corresponding average was computed from the wind tunnel data set by using the 31 gages in this region, with a standard FFT-based PSD since sufficient data were available. Figure 26 shows the points superimposed on the vehicle core. Area-weighted averages for two frequency ranges were computed, the first included all frequencies, and the second included only frequencies below the dominant frequency, as indicated in figure Fig. 25b. As mentioned earlier, the lower range was used to focus on frequencies that may excite different structural modes than the peak frequency, and excluded the effect of the large peak in the PSD.

Figure 27 presents the area-weighted average power for all frequencies for two Mach numbers at $0^{\circ}$ angle of attack. The CFD and wind tunnel data have been normalized by their respective baseline (configuration 1 , $\mathrm{M}=0.9, \alpha=0^{\circ}$ ) value. All of the canted noses, as well as the on-axis ogive nose, show significant reductions in the average power metric compared to the baseline nose, with the canted ogive showing the greatest reduction. This assessment is validated by the results of the wind tunnel data, which shows similar trends and magnitude reductions.

The same analysis was repeated for low frequencies, with the results shown in Fig. 28. Note first the scale of the ordinate axis has been changed. The data are still normalized by the baseline all-frequencies value, but the contribution from the large peak seen in the power spectral densities has been removed. At Mach 0.9, the canted noses and on-axis ogive again show a reduction compared to the baseline nose, with the canted-ogive having the largest reduction. At Mach 1.1, the reduction due to canting is minimal for these low frequencies.

Finally, for Mach 0.9 at an angle of attack of $4^{\circ}$, shown in Fig. 29a, the canted noses still show a reduction compared to the baseline; but the predicted level of the canted ogive is almost back to the baseline level, while the wind tunnel results show approximately the same reduction for all canted noses. At Mach 1.1 (Fig. 29b), the CFD shows a reduction for the canted noses; but the wind tunnel results show essentially no change. 
(a) points used for CFD cases

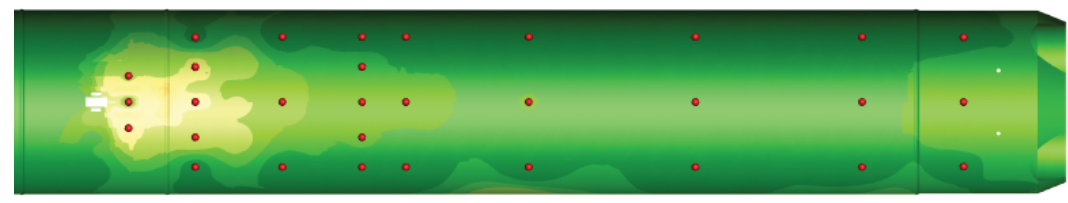

(b) points used for wind tunnel cases

Figure 26: Location of the points used to compute area-weighted average $C_{p, r m s}$.

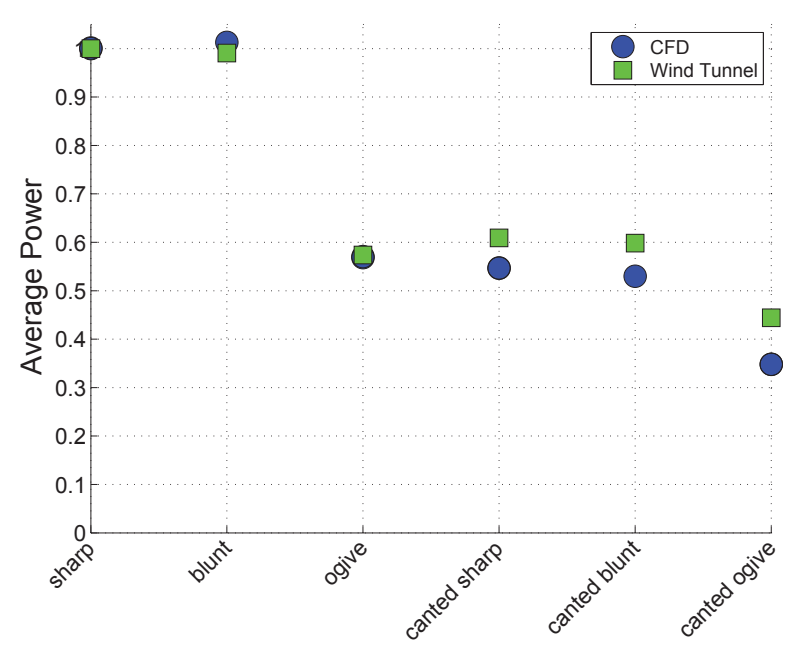

(a) $\mathrm{M}=0.9, \alpha=0^{\circ}$

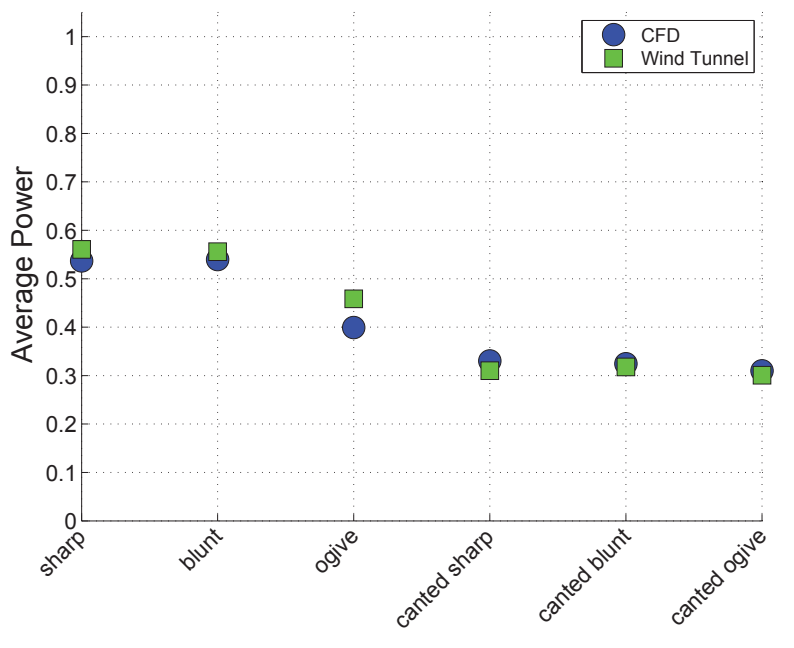

(b) $\mathrm{M}=1.1, \alpha=0^{\circ}$

Figure 27: Area-weighted average power for various booster nose shapes, all frequencies included. 


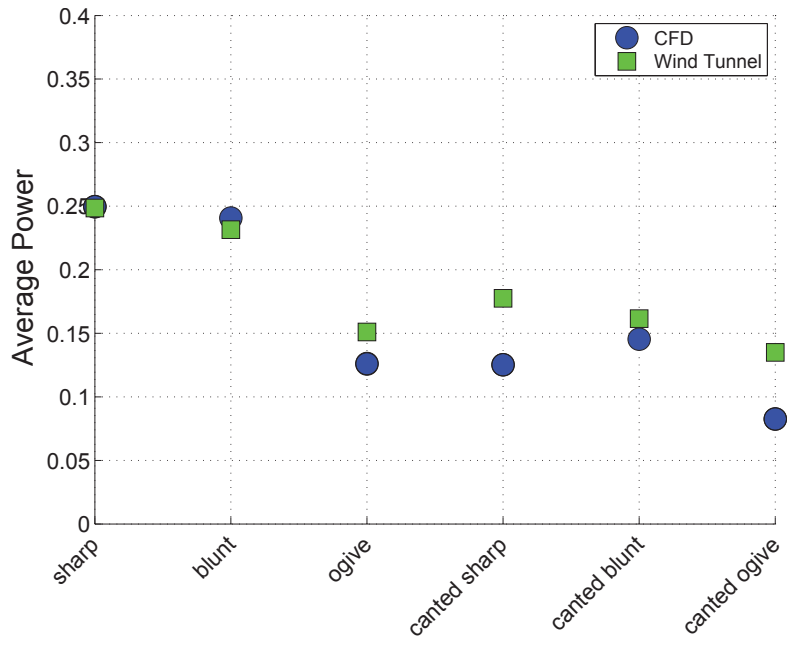

(a) $\mathrm{M}=\mathbf{0 . 9}, \alpha=0^{\circ}$

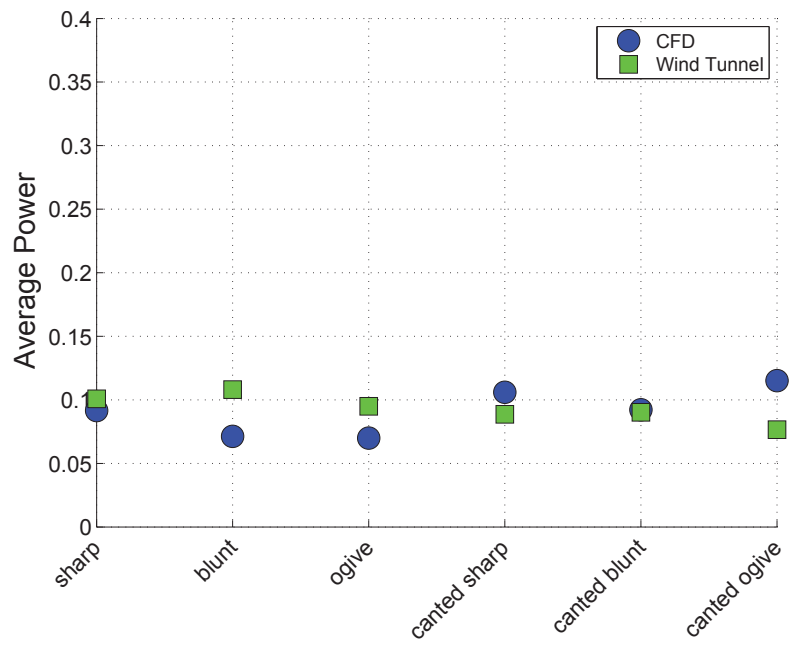

(b) $\mathrm{M}=1.1, \alpha=0^{\circ}$

Figure 28: Area-weighted average power for various booster nose shapes, low frequencies only.

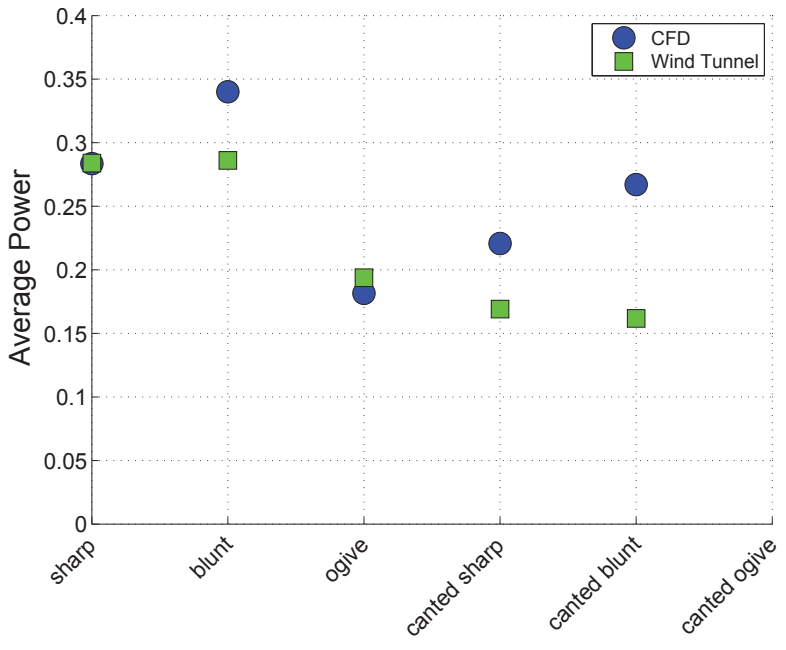

(a) $\mathrm{M}=\mathbf{0 . 9}, \alpha=4^{\circ}$

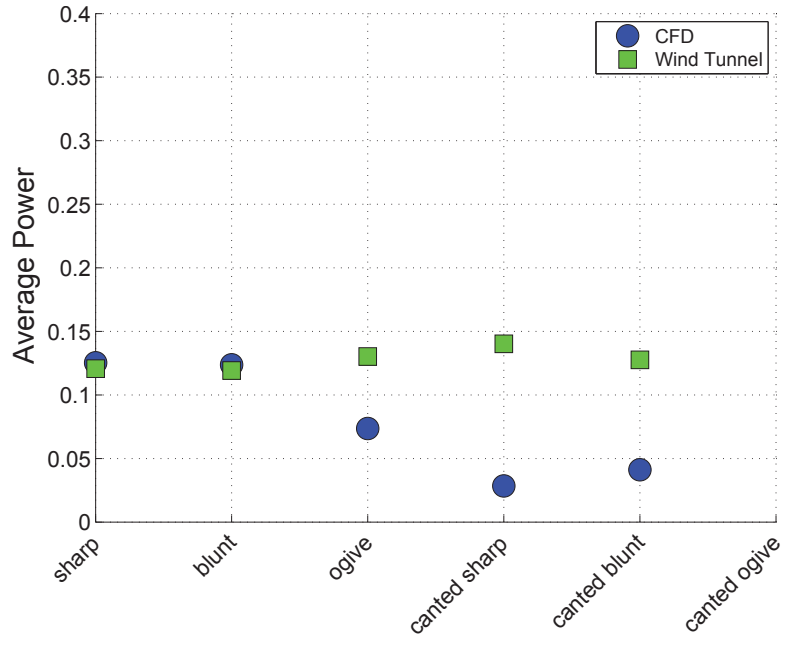

(b) $\mathrm{M}=1.1, \alpha=4^{\circ}$

Figure 29: Area-weighted average power for various booster nose shapes, low frequencies only. 


\section{Conclusions}

A computational and experimental study was undertaken to predict the time-accurate unsteady pressures on a Space Launch System (SLS) cargo configuration in the transonic flow regime, specifically in the forward booster attachment region. Comparisons were made between predictions using the FUN3D code and results from a wind tunnel test in the NASA Ames 11-ft tunnel using a 2.5\% scale model of the SLS 27000 cargo configuration. The comparisons looked at several parameters, including $C_{p, r m s}$ and power spectral densities. The peak $C_{p, r m s}$ value was under-predicted for the baseline, Mach 0.9 case, but the general trends of high $C_{p, r m s}$ levels behind the forward attach hardware, reducing as one moves away both streamwise and circumferentially, were captured. A reduction in $C_{p, r m s}$ levels at Mach 1.1 was observed for both wind tunnel and CFD. Frequency of the peak power in the power spectral density estimates was consistently under-predicted. The magnitudes of the power estimates were over-predicted in some cases, and underpredicted in others. Five alternate booster nose shapes were analyzed to demonstrate the ability of the CFD tool to predict reductions in fluctuating pressures levels due to booster nose shape. Canting the nose inward toward the core resulted in reduced $C_{p, r m s}$ levels in the forward attach region. A continuous wavelet transform (CWT) method was employed as a means to retain low frequency resolution compared to a fast Fourier transfrom (FFT) in generation of power spectral density estimates. An area-weighted average power metric based on this CWT method was used to assess and rank the various booster nose geometries, with results validated by the wind tunnel results.

\section{Appendix}

Below are the lines of MatLab ${ }^{\circledR}$ code used to generate the PSD-like output from the continuous wavelet transform (CWT). The signal is the time-varying pressure (dp) at a specific location. The wavelet name or type was morl (Morlet). The scales can be chosen in a variety of ways, and converted to approximate frequencies (fpseudo) by using the scl2frq function and the time increment ( $\mathrm{dt}$ ). The CWT uses the scales, not the pseudo-frequencies. The power at each scale (cwtpf) was determined as a ratio to the total power, then scaled by the RMS value of the original signal. The corresponding FFT-based PSD was generated using the pwelch command. The same signal is used. The window size (mw) depends on the frequency resolution one desires and the percentage overlap (we used $75 \%$ ). The sampling rate (fs) is the inverse of the time step.

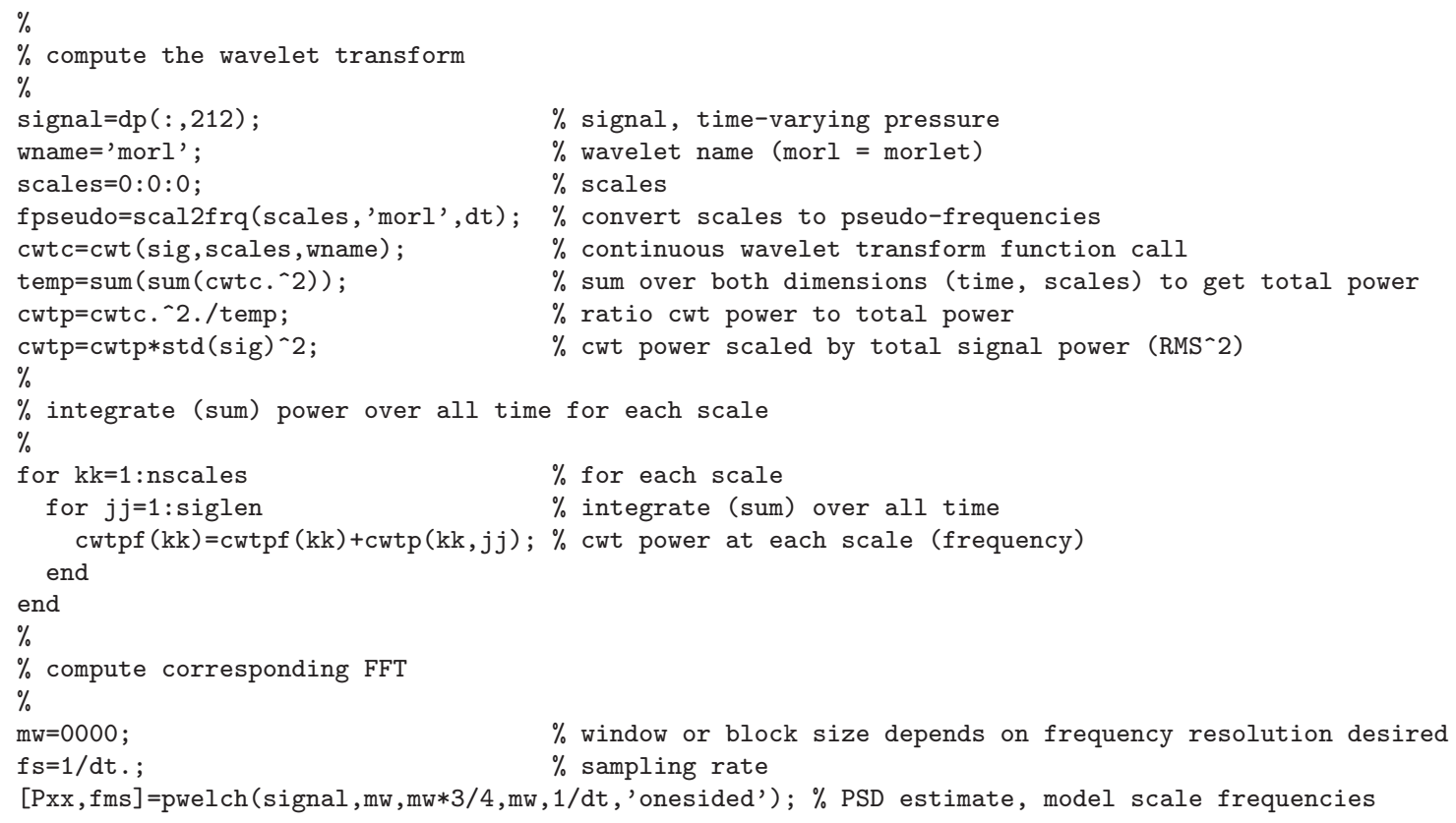




\section{Acknowledgments}

The authors would like to thank John Blevins and Darren Reed of the NASA Marshall Space Flight Center. John was responsible for the aerodynamic environments for SLS, and graciously allowed the piggyback arrangement on the very important mainline SLS wind tunnel test. Darren was the lead engineer for the mainline test, and worked with us to ensure we obtained the data needed to validate the computation results.

\section{References}

${ }^{1}$ Singer, J. A., Cook, J. R., and Singer, C. E., "NASA Space Launch System Operations Strategy," AIAA Paper 2012$15221,2012$.

${ }^{2}$ NASA, "NASA Space Launch System (SLS)," http://en.wikipedia.org/wiki/Space_Launch_System, [last accessed 16 October 2014].

${ }^{3}$ Cole, H. A., Erickson, A. L., and Rainey, A. G., "Buffeting During Atmospheric Ascent - Space Vehicle Design Criteria," NASA SP 8001 (Rev), National Aeronautics and Space Administration, 1970.

${ }^{4}$ Piatak, D. J., Sekula, M. K., and Rausch, R. D., "Ares Launch Vehicle Transonic Buffet Testing and Analysis Techniques," Journal of Spacecraft and Rockets, Vol. 49, No. 5, 2012, pp. 798-807.

${ }^{5}$ Piatak, D. J., Sekula, M. K., and Rausch, R. D., "Comparison of Ares I-X Wind-Tunnel-Derived Buffet Environment with Flight Data," Journal of Spacecraft and Rockets, Vol. 49, No. 5, 2012, pp. 822-833.

${ }^{6}$ Engblom, W. A., "Numerical Simulation of Titan IVB Transonic Buffet Environment," Journal of Spacecraft and Rockets, Vol. 40, No. 5, 2003, pp. 648-656.

${ }^{7}$ Pain, R., Weiss, P. E., and Deck, S., "Zonal Detached Eddy Simulation of the Flow Around a Simplified Launcher Afterbody," AIAA Journal, Vol. 52, No. 9, 2014, pp. 1967-1979.

${ }^{8}$ Alter, S. J., Brauckmann, G. J., Kleb, B., Streett, C. L., Glass, C. E., and Schuster, D. M., "Time Accurate Unsteady Pressure Loads Simulated for the Space Launch System at a Wind Tunnel Condition," AIAA Paper (to be published), 2015.

${ }^{9}$ Biedron, R. T., Derlaga, J. M., Park, M. A., Hammond, D. P., Gnoffo, P. A., Carlson, J. R., Thomas, J. L., Lee-Rausch, E. M., White, J. A., Carpenter, M. H., Jones, W. T., Runsey, C. L., Vatsa, V. N., Derlaga, J. M., Wood, W. A., Kleb, W. L., O'Brien, Jr., D. M., Smith, M. J., and Lynch, C. E., "FUN3D Manual: 12.4," NASA TM 2014-218179, March 2014.

${ }^{10}$ Vatsa, V. N., Lockard, D. P., and Khorrami, M. R., "Application of FUN3D Solver for Aeroacoustics Simulation of a Nose Landing Gear Configuration," AIAA Paper 2011-2820, 2011.

${ }^{11}$ Edwards, J. R., "A Low-Diffusion Flux-Splitting Scheme for Navier-Stokes Calculation," Computers and Fluids, Vol. 26, No. 6, 1997, pp. 653-659.

${ }^{12}$ van Albada, G. D., van Leer, B., and Roberts, W. W., "A comparative Study of Computational Methods in Cosmic Gas Dynamics," Astronomy and Astrophysics, Vol. 108, No. 1, 1982, pp. 76-84.

${ }^{13}$ Vatsa, V. N. and Lockard, D. P., "Assessment of Hybrid RANS/LES Turbulence Models for Aeroacoustics Assessment of Hybrid RANS/LES Turbulence Models for Aeroacoustics Applications," AIAA Paper 2010-4001, 2010.

${ }^{14}$ Vatsa, V. N., Carpenter, M. H., and Lockard, D. P., "Re-evaluation of an Optimized Second Order Backward Difference (BDF2OPT) Scheme for Unsteady Flow Applications," AIAA Paper 2010-0122, 2010.

${ }^{15}$ Biswas, R., Dunbar, J. A., Hardman, J. P., Bailey, F. R., Wheeler, L. F., and Rogers, S. E., "The Impact of High-End Computing on NASA Missions," IT Professional, Vol. 14, No. 2, 2012, pp. 20-28.

${ }^{16} \mathrm{NASA}$, http://www.nasa.gov/centers/ames/orgs/aeronautics/windtunnels/11x11-wind-tunnel.html, [last accessed 10 October 2014].

${ }^{17}$ Kmak, F., "Modernization and Activation of the NASA Ames 11-By 11-Foot Transonic Wind Tunnel," AIAA Paper 2000-2680, 2000.

${ }^{18}$ Chavalier, H. L. and Robertson, J. E., "Pressure Fluctuations Resulting from an Alternating Flow Separation and Attachment at Transonic Speeds," AEDC TDR 63-204, November 1963.

${ }^{19}$ Welch, P. D., "The Use of Fast Fourier Transform for the Estimation of Power Spectra: A Method Based on Time Averaging Over Short, Modified Periodograms," IEEE Trans. Audio Electroacoustics, Vol. AU-15, 1967, pp. 70-73.

${ }^{20}$ Daubechies, I., "The Wavelet Transform, Time-Frequency Localization and Signal Analysis," IEEE, Vol. 36, No. 5, 1990, pp. 961-1004.

${ }^{21}$ Mathworks, http://www.mathworks.com/help/wavelet/gs/continuous-wavelet-transform.html, [last accessed 10 October 2014]. 